Pacific Northwest

National Laboratory

Operated by Battelle for the

U.S. Department of Energy

\section{Underwater Determination of Radionuclide Levels in 105-KE Basin Floor and Walls Using a Gamma-Ray Detector System}

\author{
R. J. Arthur \\ S. N. Schlahta \\ R. I. Scherpelz \\ P. J. MacFarlan \\ M. A. Catalan
}

September 2003

Prepared for the U.S. Department of Energy under Contract DE-AC06-76RL01830 


\title{
DISCLAIMER
}

This report was prepared as an account of work sponsored by an agency of the United States Government. Neither the United States Government nor any agency thereof, nor Battelle Memorial Institute, nor any of their employees, makes any warranty, express or implied, or assumes any legal liability or responsibility for the accuracy, completeness, or usefulness of any information, apparatus, product, or process disclosed, or represents that its use would not infringe privately owned rights. Reference herein to any specific commercial product, process, or service by trade name, trademark, manufacturer, or otherwise does not necessarily constitute or imply its endorsement, recommendation, or favoring by the United States Government or any agency thereof, or Battelle Memorial Institute. The views and opinions of authors expressed herein do not necessarily state or reflect those of the United States Government or any agency thereof.

\author{
PACIFIC NORTHWEST NATIONAL LABORATORY \\ operated by \\ BATTELLE \\ for the \\ UNITED STATES DEPARTMENT OF ENERGY \\ under Contract DE-AC06-76RL01830
}

This document was printed on recycled paper. 


\title{
Underwater Determination of Radionuclide Levels in 105-KE Basin Floor and Walls Using a Gamma-Ray Detector System
}

\author{
R. J. Arthur \\ S. N. Schlahta \\ R. I. Scherpelz \\ P. J. MacFarlan \\ M. A. Catalan
}

September 2003

Prepared for the U.S. Department of Energy

under Contract DE-AC06-76RL01830

Pacific Northwest National Laboratory

Richland, Washington 99352 


\section{Summary}

The $105 \mathrm{~K}$ East Basin was originally built to store spent nuclear fuel from the Hanford K East Reactor before processing the fuel in the Plutonium-Uranium Extraction facility. In the 1970s and later years, the basin was used for longer-term storage of $\mathrm{N}$ Reactor fuel, which is still the primary content of the basin today. The basin is divided into three bays by two concrete partition walls and contains various pits as well as a discharge-chute area adjacent to the K East Reactor. Each basin contains water nearly 17 feet in depth and has a wetted area of concrete floor and walls of approximately $26,500 \mathrm{ft}^{2}$ (i.e., floor $9,900 \mathrm{ft}^{2}$ and walls $16,600 \mathrm{ft}^{2}$ ). Currently, sand filter and ion exchange modules provide pathways to remove particulate and contaminants from the water. The K East Basin concrete floor and walls are primarily uncoated. Exceptions are 1) an approximately 1.5-ft band of concrete running around the current basin waterline, which has been wire brushed and coated with epoxy (Huang and Moore 1997) as part of the dose-reduction activities in 1995 (with the water level of the basin subsequently raised approximately 1 foot to its current level) and 2) the Discharge Chute area and portions of the Tech View Pit, which reportedly have been painted (Vargo et al. 1994).

Activities to remove spent fuel, sludge, and debris from K East Basin have begun. Once all the radioactive hazards (i.e., fuel, sludge, and debris) are removed from the basin, the basin water will be removed, and the basin will be turned over to a deactivation and decommission contractor. However, the specific approach to achieving this end state is dependent on knowledge of contamination levels in the concrete walls and floor once the water is removed. The Spent Nuclear Fuel Project contracted ${ }^{(a)}$ with the Pacific Northwest National Laboratory (PNNL) to perform nondestructive evaluation of the K East Basin for the purpose of determining concrete contamination levels, including depth of penetration, from selected wall and floor locations below the basin waterline. The definition of the initial requirements and specific approach were controlled through a Data Quality Objectives (DQO) and Sample Analysis Plan (SAP) methodology managed by the Spent Nuclear Fuel Project (Makenas 2002, Baker et al. 2002). This initial approach sought to maximize the useful data from these analyses to make decisions related to final disposition of the K Basins.

The $\mathrm{K}$ East Basin presents the greatest challenge from the perspective of decontamination for the Spent Nuclear Fuel Project. This is because K East Basin has been in service, storing N reactor fuel, longer than K West Basin (i.e., 1975 versus 1981) and, unlike K West Basin, K East Basin walls and floors were not cleaned and coated with epoxy before entering this type of service. K East Basin also has the greater amount of fuel-bearing sludge in contact with the floor and walls (Baker 1995; Baker 2001). The fact that canisters of fuel in K East Basin do not have lids and often have perforated bottoms (again, unlike in K West Basin), and the more checkered history of water-quality maintenance in K East Basin (Johnson and Burke 1995) also add to the potential for higher radioactive contamination of the $\mathrm{K}$ East Basin walls and floor. Thus, the characterization of the $\mathrm{K}$ East Basin is expected to exceed the level of contamination that might be found in $\mathrm{K}$ West Basin, thus providing an upper bound to overall basin contamination.

The PNNL team designed, fabricated, tested, and deployed two separate detector systems to measure basin contamination levels consistent with direction of the DQO and SAP. One system was developed to measure the contamination levels on the basin walls and the other to measure the contamination levels on the floor. The wall detector system was taken out of operation after the submersible vessel protecting the detector from the basin water leaked. It was not possible to repair the wall detector system due to two primary factors: 1) the risk of radioactive exposure to staff involved in the decontamination and repair of

(a) Contract 11979-79, "Nondestructive Examination of the Depth of Contamination in the Walls and Floor of the K East Basin, from FH to PNNL, transmitted February 22, 2002.

Contract 16282-12, "Nondestructive Examination of the Depth of Contamination in the Walls and Floor of the K East Basin, from FH to PNNL, transmitted September 16, 2002. 
the wall detector system and 2) the impact of the repair activity to ongoing basin operations as the potential for airborne contamination would result in measures that would impede other basin activities. The floor detector was deployed successfully. Measurements were taken at seven floor locations in either the East or West Bay. In most cases, a single location provided several discrete measurements (i.e., after a low-pressure wash, after a high-pressure wash, and after a stainless-steel brushing). No measurements were taken from the Center Bay. To take measurements from the desired Center Bay locations, several fuel moves would be necessary to minimize the influence of the spent fuel on the measurements. Basin resources were not available to perform the needed fuel moves. Due to the wall detector system being unavailable, special equipment was designed to enable the floor-detector system to take one wall measurement.

Prior to the current measurements, the underwater surfaces of the basin were assumed to be primarily contaminated with ${ }^{137} \mathrm{Cs}$ at levels near $12.9 \mu \mathrm{Ci} / \mathrm{cm}^{2}$, as found on the 1980 core samples (Bechtold 1981) from the basin walls near the water surface. This was the only data available and it was acknowledged that additional measurements might differ significantly from these very limited samples. The floor detector system measured ${ }^{137} \mathrm{Cs}$ activity levels significantly higher than expected. Activity levels of ${ }^{137} \mathrm{Cs}$ on the basin floor ranged from a low of $85 \mu \mathrm{Ci} / \mathrm{cm}^{2}$ to a high $3800 \mu \mathrm{Ci} / \mathrm{cm}^{2}$, depending on location. There appeared to be only modest benefit by attempts to clean the floor using high-pressure water followed with a stainless steel brush. Activity levels of ${ }^{137} \mathrm{Cs}$ levels on the basin wall before cleaning were 360 to 368 $\mu \mathrm{Ci} / \mathrm{cm}^{2}$ and $176 \mu \mathrm{Ci} / \mathrm{cm}^{2}$ after cleaning with a stainless steel brush. Nonetheless, the activity level at each location, whether on the floor or wall, was too high for a depth-of-penetration analysis because the surface activity dominated the data spectrum and far exceeded the activity level used to calibrate the system. A post-campaign calibration was performed, confirming that the system was still functioning properly.

It is worth noting that there was considerable variation in the activity levels measured from one bay of the basin to another, from one cubicle to another in the same bay, or from the west side of a cubicle to the east side of the same cubicle. The highest and lowest activity levels measured were found in the same cubicle. The activity level was not significantly reduced using either the high-pressure wash or stainless steel brushing, which is possibly due to radionuclide contamination embedded into the concrete or sludge particles remaining in the pores of the concrete. It will take more aggressive cleaning or remediation techniques than were used in this work to effectively reduce the contamination on and in the basin concrete.

\section{References}

Baker RB. 1995. Summary Status of K Basins Sludge Characterization. WHC-SD-SNF-TI-006, Westinghouse Hanford Company, Richland, WA.

Baker RB. 2001. Estimated Volumes of Sludge in the Hanford K East and K West Basins. HNF-8118, Rev. 0, Fluor Hanford, Richland, WA.

Bechtold DB. 1981. KE Fuel Storage Facility Activity Mapping in Support of Exposure Reduction. UNI-1697, United Nuclear Industries, Richland, WA.

Huang FF, and FW Moore. 1997. Dose Reduction Improvements in Storage Basins of Spent Nuclear Fuel. HNF-SA-3158-FP, Fluor Daniel Northwest, Richland, WA.

Johnson AB, and S Burke. 1995. K Basin Corrosion Program Report. WHC-EP-0877, Westinghouse Hanford Company, Richland, WA. 
Vargo GJ, JS Durham, EE Hickey, PS Stansbury, and GR Cicotte. 1994. Review of ALARA Plan for Activities at the 105 K-East Fuel Storage Basin. PNL-9826, Rev. 2, Pacific Northwest Laboratory, Richland WA. 



\section{Acronyms}

$\begin{array}{ll}\text { ADC } & \text { analog digital converter } \\ \text { DQO } & \text { Data Quality Objectives } \\ \text { FH } & \text { Fluor Hanford } \\ \text { FWHM } & \text { full-width at half-maximum } \\ \text { HPGe } & \text { high-purity germanium detector } \\ \text { MAC } & \text { multi-attitude cryostat } \\ \text { MCA } & \text { multi-channel analyzer } \\ \text { MCNP } & \text { Monte Carlo N-Particle Transport Code } \\ \text { NDE } & \text { nondestructive evaluation } \\ \text { NIM } & \text { nuclear instrumentation module } \\ \text { NIST } & \text { National Institute of Science and Technology } \\ \text { PUREX } & \text { Plutonium-Uranium Extraction } \\ \text { PNNL } & \text { Pacific Northwest National Laboratory } \\ \text { PVC } & \text { polyvinyl chloride } \\ \text { SAP } & \text { Sampling and Analysis Plan } \\ \text { SNF } & \text { spent nuclear fuel } \\ \text { SNFP } & \text { Spent Nuclear Fuel Project } \\ \text { SYNTH } & \text { Gamma-ray Spectrum Synthesizer Code } \\ & \end{array}$





\section{Contents}

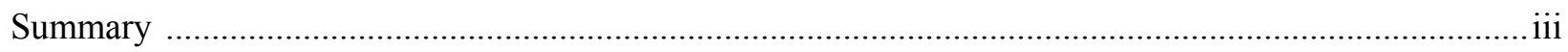

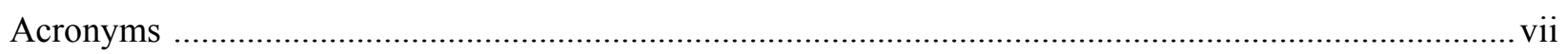

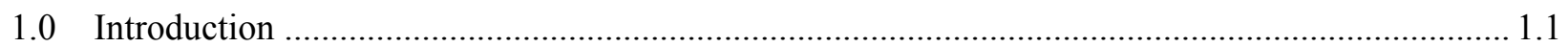

2.0 Basis for the Technical Approach .......................................................................................... 2.1

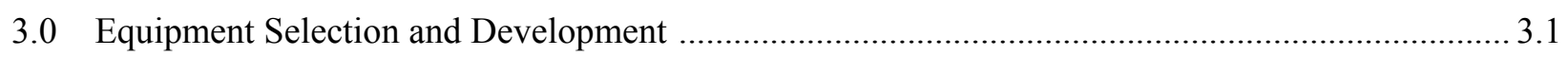

3.1 Canberra High-Purity Germanium Detector ….......................................................... 3.1

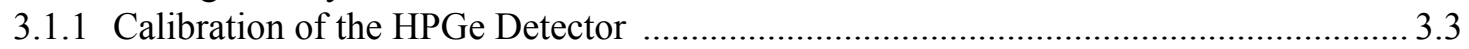

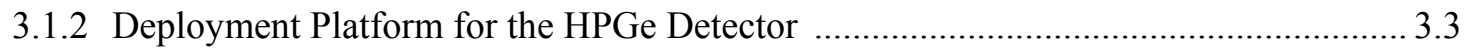

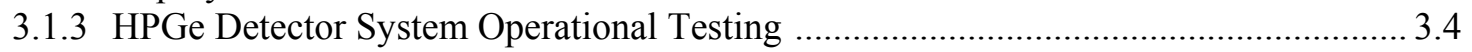

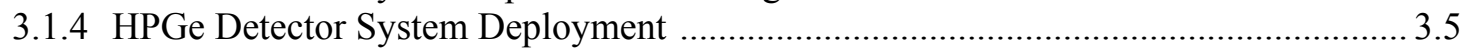

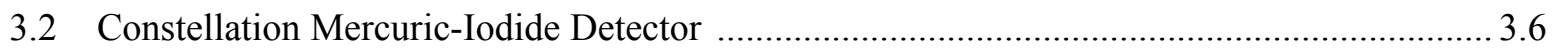

3.2.1 Mercuric-Iodide-Detector Deployment Platform ..................................................... 3.9

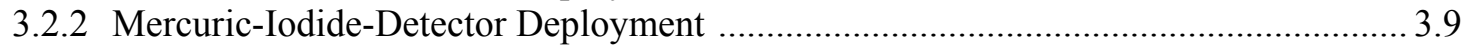

3.2.3 Calibration of the Mercuric-Iodide Detector .............................................................. 3.14

3.2.4 Penetration Calibration Using Mercuric-Iodide Detector ........................................ 3.14

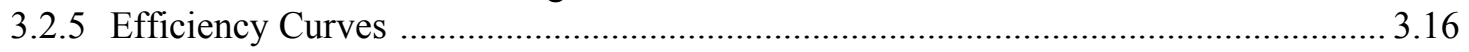

3.2.6 Validation Methods and Traceability of Sources ................................................... 3.17

3.2.7 Experimental Data Used to Determine the Distance between Floor

Measurement Locations and Spent Fuel ……..................................................... 3.18

3.2.8 Activity Levels Determined via Gamma-Ray Spectrometry .................................... 3.18

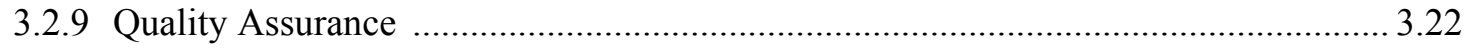

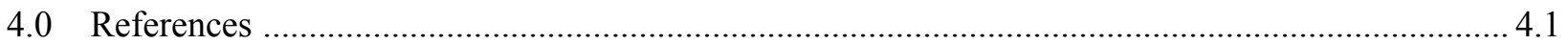

Appendix A: Manufacturer Specification for HPGe Detector

Appendix B: Sketch of HPGe Detector System Submersible Vessel

Appendix C: Manufacturer Specification for $\mathrm{HgI}_{2}$ Detector

Appendix D: Sketch of $\mathrm{HgI}_{2}$ Detector System Submersible Vessel 


\section{Figures}

1.1. Overview Schematic of K West (and East) Basin 1.1

3.1. Canberra Model GC3518 Detector with a "Big MAC" Cryostat 3.1

3.2. ANTEC Submersible Vessel Upon Delivery..... 3.2

3.3. HPGe Detector System Testing in 306 East 3.5

3.4. Constellation Technology Mercury-Detector Module ............................................................... 3.6

3.5. Cut Away Drawing of the $\mathrm{HgI}_{2}$ Submersible Vessel ................................................................. 3.7

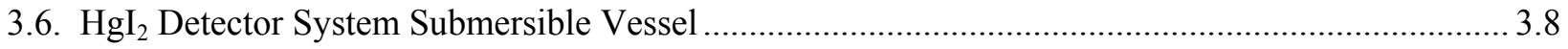

3.7. Sludge Removal—Slotted Canister Set and Spray Wand........................................................ 3.10

3.8. Sludge-Removal Effectiveness Testing in 306 East .............................................................. 3.11

3.9. Video Picture of Cubicle 2209 West After 110 psig Wash ......................................................... 3.11

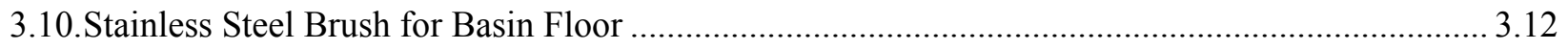

3.11.Table Designed to Take Wall Measurement Using $\mathrm{HgI}_{2}$ Detector System .................................. 3.12

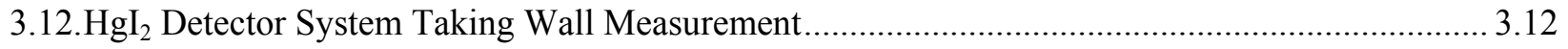

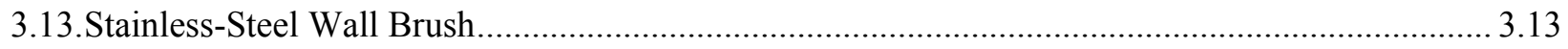

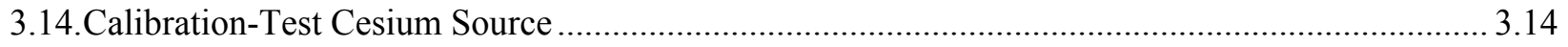

3.15.Effects of 42.5-mm Thickness of Concrete on Gamma Rays Deposited in the $\mathrm{HgI}_{2}$ Detector

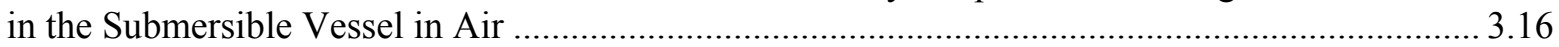

3.16. Graphical Display of the Effects on Data Quality as the Floor Detector is Moved Close to Canisters Containing Spent Fuel................................................................................................. 3.19

3.17. Graphical Display of All Significant Data in the Data-Collection Campaign for the K East Basin Floor. 


\section{Tables}

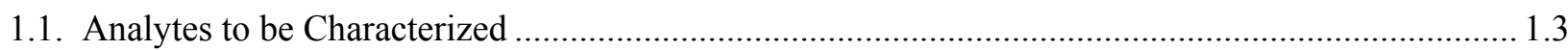

2.1. Ratios for Full-Energy to Valley Count Rates ............................................................................. 2.1

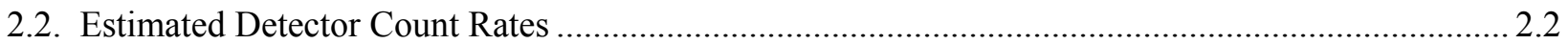

3.1. HPGe Detector in Submersible Vessel in Air with 3/4-inch Tungsten Collimator in Front of Detector $\left(2.03 \mu \mathrm{Ci}\right.$ large-area ${ }^{137} \mathrm{Cs}$ source $)$.......................................................................... 3.3

3.2. Mercuric-Iodide Detector in Submersible Vessel in Air in Front of Detector

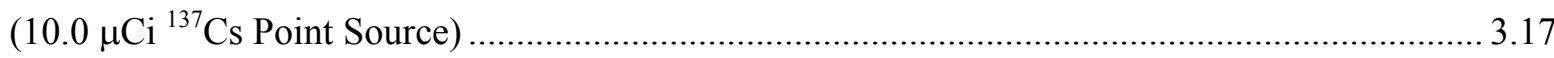

3.3. Summary of NDE Measurements on KE Basin Floor and Wall ................................................ 3.20 


\subsection{Introduction}

The $105 \mathrm{~K}$ East Basin was originally built to accommodate storage of spent nuclear fuel (SNF) from the Hanford K East Reactor before processing the fuel in the Plutonium-Uranium Extraction (PUREX) facility. In the 1970s and later years, the basin was used for longer-term storage of N Reactor fuel, which is still the primary content of the basin today. The basin is divided into three bays by two concrete partition walls and contains various pits as well as a discharge chute area adjacent to the K East Reactor (Figure 1.1). Each basin contains water nearly 17 feet in depth and has a wetted area of concrete floor and walls of approximately 26,500 $\mathrm{ft}^{2}$ (i.e., floor 9,900 $\mathrm{ft}^{2}$ and walls $16,600 \mathrm{ft}^{2}$ ). Currently, sand-filter and ion exchange modules provide pathways to remove particulates and contaminants from the water. The $\mathrm{K}$ East Basin concrete floor and walls are primarily uncoated. Exceptions are 1) an approximately 1.5-ft band of concrete running around the current basin waterline, which has been wire brushed and coated with epoxy (Huang and Moore 1997) as part of the dose-reduction activities in 1995 (with the water level of the basin subsequently raised approximately 1 foot to its current level) and 2) the Discharge Chute area and portions of the Tech View Pit, which reportedly have been painted (Vargo et al. 1994).

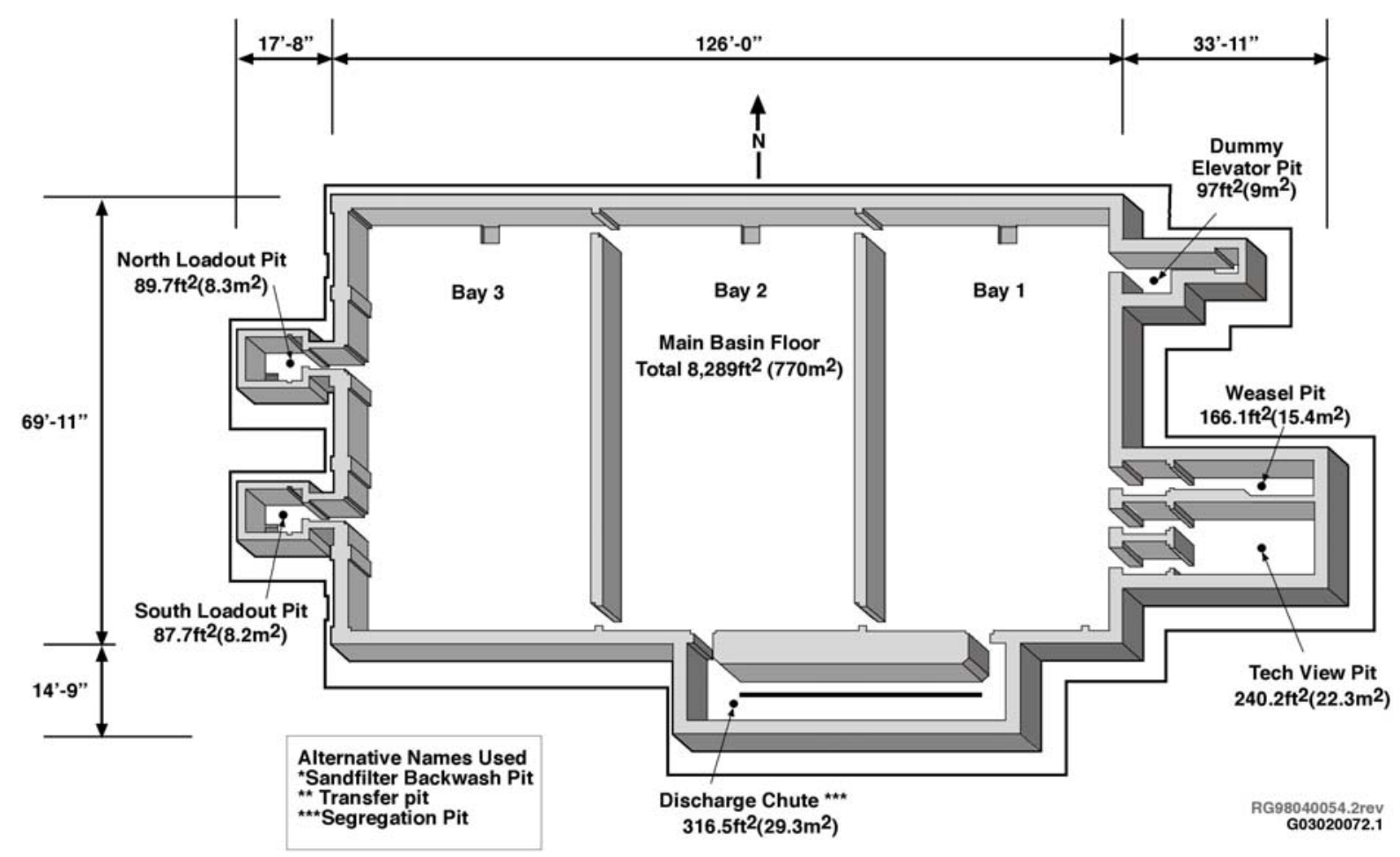

Figure 1.1. Overview Schematic of K West (and East) Basin

Activities to remove spent fuel, sludge, and debris from K East Basin have begun. Once all the radioactive hazards (i.e., fuel, sludge, and debris) are removed from the basin, the water will be removed, and the basin will be deactivated and decommissioned. However, the specific approach to achieving this end state is dependent on knowledge of contamination levels in the concrete walls and floor once the water is removed. The Spent Nuclear Fuel Project contracted with the Pacific Northwest National 
Laboratory (PNNL) to perform nondestructive evaluation (NDE) of the K East Basin for the purpose of determining concrete contamination levels, including depth of penetration, from selected wall and floor locations below the basin waterline.

The K East Basin presents the greatest challenge from the perspective of decontamination for the Spent Nuclear Fuel Project. This is because K East Basin has been in service, storing N reactor fuel, longer than K West Basin (i.e., 1975 versus 1981) and, unlike K West Basin, K East Basin walls and floors were not cleaned and coated with epoxy before entering this type of service. K East Basin also has the greater amount of fuel-bearing sludge in contact with the floor and walls (Baker 1995; Baker 2001). The fact that canisters of fuel in $\mathrm{K}$ East Basin do not have lids and often have perforated bottoms (again, unlike in $\mathrm{K}$ West Basin), and the more checkered history of water-quality maintenance in K East Basin (Johnson and Burke 1995) also add to the potential for higher radioactive contamination of the K East Basin walls and floor. Thus, the characterization of the $\mathrm{K}$ East Basin is expected to exceed the level of contamination that might be found in $\mathrm{K}$ West Basin.

This report documents the process and results associated with the execution of the Fluor Hanford (FH) Statements of Work (Contracts 11979-78 and 16282-12) (a) $^{(\mathrm{a})}$ authorizing PNNL to survey the K East Basin for radionuclide contamination on and in the concrete walls and floor using NDE techniques. The data contained in this report supported an FY 2003 decision by the Spent Nuclear Fuel Project to determine the method(s) to be applied in deactivating the basin once fuel and sludge have been removed. A technical data package containing the standard operating procedure for taking floor measurements, log sheets, data files, and spectra for each measurement location is available from FH (R. B. Baker).

The strategy for characterizing the contamination levels in the K East Basin concrete walls and floor was formulated and controlled by following a specific methodology managed by the Spent Nuclear Fuel Project based on Data Quality Objectives (DQO) (Makenas and Baker 2002) and Sampling and Analysis Plan (SAP) (Baker et al. 2002). The approach focused on implementing two complementary characterization techniques: 1) an in situ NDE approach and 2) a limited sampling of the concrete walls in the form of retrieved cores that would be analyzed. It was intended that the NDE method would provide the ability to determine the contamination levels, including the depth of contamination, in many locations in the basin (e.g., floors, supporting walls) with scoping accuracy; while the core samples would provide a limited number of detailed, but more accurate, data from selected non-supporting basin walls. The subject DQO and SAP expected that the core samples would be used to validate and calibrate the NDE measurements. The primary objectives of the NDE characterization effort as described in the DQO and SAP documents were:

1. To acquire data on the depth of radionuclide penetration (radionuclides of concern are listed in Table 1.1) in the basin walls/floor to determine possible radiation-dose rates in the basin structure and the immediate surroundings once the basin water level is lowered and ultimately removed.

(a) Contract 11979-79, "Nondestructive Examination of the Depth of Contamination in the Walls and Floor of the K East Basin, from FH to PNNL, transmitted February 22, 2002.

Contract 16282-12, "Nondestructive Examination of the Depth of Contamination in the Walls and Floor of the K East Basin, from FH to PNNL, transmitted September 16, 2002. 
Table 1.1. Analytes to be Characterized

\begin{tabular}{|c|c|c|c|}
\hline $\begin{array}{l}\text { Isotope or } \\
\text { Compound }\end{array}$ & $\begin{array}{c}\text { For Coring } \\
\text { Samples? } \\
{[\mathrm{Y} / \mathrm{N}]} \\
\end{array}$ & $\begin{array}{c}\text { For NDE (Gamma } \\
\text { Scan) Measurements? } \\
{[\mathrm{Y} / \mathrm{N}]} \\
\end{array}$ & Remarks \\
\hline${ }^{137} \mathrm{Cs},{ }^{134} \mathrm{Cs}$ & Y & 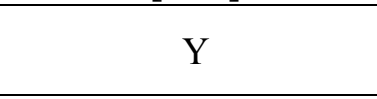 & $\begin{array}{l}\text { Expected to be primary } \\
\text { penetrating species. }\end{array}$ \\
\hline${ }^{60} \mathrm{Co}$ & Y & Y & \\
\hline${ }^{152} \mathrm{Eu},{ }^{154} \mathrm{Eu},{ }^{155} \mathrm{Eu}$ & $\mathrm{Y}$ & $\mathrm{Y}$ & $\begin{array}{l}\text { Low concentration but high- } \\
\text { energy gamma emission. }\end{array}$ \\
\hline $\begin{array}{l}{ }^{212} \mathrm{Bi},{ }^{208} \mathrm{Tl},{ }^{125} \mathrm{Sb}, \\
{ }^{226} \mathrm{Ra},{ }^{95} \mathrm{Nb}, \\
{ }^{106} \mathrm{Ru} / \mathrm{Rh},{ }^{144} \mathrm{Ce} / \mathrm{Pr}\end{array}$ & $\mathrm{Y}$ & $\mathrm{Y}$ & \\
\hline${ }^{90} \mathrm{Sr}$ & $\mathrm{Y}$ & $\mathrm{Y}$ & $\begin{array}{l}\text { NDE may detect X-rays from } \\
\text { Beta interactions with concrete. }\end{array}$ \\
\hline PCBs & $\mathrm{Y}$ & $\mathrm{N}$ & \\
\hline${ }^{241} \mathrm{Am}$ & $\mathrm{Y}$ & $\mathrm{Y}$ & \\
\hline $\begin{array}{l}\mathrm{U} \text { total and } \mathrm{U} \\
\text { isotopics }\end{array}$ & $\mathrm{Y}$ & $\mathrm{N}$ & \\
\hline $\begin{array}{l}239 / 240 \mathrm{Pu},{ }^{238} \mathrm{Pu}, \\
{ }^{243 / 244} \mathrm{Cm},{ }^{237} \mathrm{~Np}\end{array}$ & $\mathrm{Y}$ & $\mathrm{N}$ & \\
\hline $\begin{array}{l}\text { Gross Alpha and } \\
\text { Beta }\end{array}$ & $\mathrm{Y}$ & $\mathrm{N}$ & \\
\hline${ }^{99} \mathrm{Tc},{ }^{3} \mathrm{H},{ }^{14} \mathrm{C}$ & $\mathrm{Y}$ & $\mathrm{N}$ & \\
\hline
\end{tabular}

2. To acquire data on the distribution and nature of the radiological contamination in the walls/floor to help determine which methods can be realistically used to remove/reduce the radiological dose/contamination as well as to determine which areas are most in need of mitigation.

Due to the unique technical challenges as well as a very tight schedule, the PNNL contract with FH included a hold point where PNNL staff were to develop and present a proposed plan for conducting the NDE activity to a FH senior review panel. FH established the review panel of Spent Nuclear Fuel Project (SNFP) Transition Projects staff and other senior FH staff to confirm the feasibility and approach being proposed. On March 22, 2002, PNNL presented its plan for the NDE analysis of K East Basin walls and floor. The SNFP Transitions Project panel determined that the PNNL plan for NDE met the DQO and SAP requirements ${ }^{(a)}$ (Baker et al. 2002). The following is a summary of the proposed PNNL plan:

- Wall measurements to be taken at over 100 locations in the basin, including triplicate measurements at the likely core sampling locations.

- Up to five measurements to be taken from the basin floor.

- Measurements to be limited to the main basin.

(a) Internal memo from R. B. Baker to R. M. Suyama, FH, "Holdpoint Review for PNNL Plan for Nondestructive Evaluation of Contamination Penetration into the Walls and Floors of K East Basin," 02-SNF/RBB-001, transmitted March 27, 2002. 
- Two different gamma spectrometers used to obtain the NDE measurements: high-purity germanium detector (HPGe) for wall measurements and a mercuric-iodide $\left(\mathrm{HgI}_{2}\right)$ detector for floor measurements.

- Measurements will be interpreted to provide depth of penetration (i.e., concentration as a function of depth) through modeling with the Monte Carlo N-Particle Transport Code (MCNP) and calibrations to standards (Briesmeister 2000).

- Uncertainties in measurements are difficult to estimate and relatively large. An estimate would be assuming exponential decrease in contamination by depth and that there would be an estimated 50\% uncertainty in discriminating between 3/16 inch and 3/8 inch; and an estimated 10-25\% uncertainty for depths greater than $1 / 2$ inch. It was determined that the large uncertainty was acceptable.

- Schedule was very aggressive for completing the design, fabrication, and preparation to deploy both detector systems (approximately 12 weeks in total duration). 


\subsection{Basis for the Technical Approach}

Gamma rays emitted by radioactive decay of ${ }^{137} \mathrm{Cs}$ have an initial energy of $662 \mathrm{keV}$. In reality, ${ }^{137} \mathrm{Cs}$ decays into ${ }^{137 \mathrm{~m}} \mathrm{Ba}$. ${ }^{137 \mathrm{~m}} \mathrm{Ba}$ has a half-life of 2.55 minutes versus 30.17 years for ${ }^{137} \mathrm{Cs}$, guaranteeing that they are in radioactive equilibrium. When the ${ }^{137 \mathrm{~m}} \mathrm{Ba}$ decays, it emits a $662-\mathrm{keV}$ gamma, and this gamma emission is usually identified with the presence of ${ }^{137} \mathrm{Cs}$. A $662-\mathrm{keV}$ gamma emitted by a ${ }^{137 \mathrm{~m}} \mathrm{Ba}$ atom that enters the detector crystal without any interactions along the way will have a high probability of depositing its full energy of $662 \mathrm{keV}$ in the detector crystal, resulting in a count in the "full energy peak" of the collected spectrum. However, if this gamma experiences a scattering interaction with another atom before entering the crystal, it will lose some of its energy in the interaction and deposit less than the full energy of $662 \mathrm{keV}$ in the crystal. The relative frequency of counts in the multiple-scatter region of the gamma spectrum compared to the counts in the full-energy peak is thus an indication of the amount of concrete (i.e., in terms of thickness) between the radioisotopes that emit gammas and the detector.

Understanding the contribution of multiple-scattered gammas to the overall spectrum, and interpreting this portion of a measured spectrum, would rely heavily on modeling and laboratory measurements. Radiation-transport computer codes, such as MCNP, have been used to model the interaction of radiation with materials and the subsequent detector response. The PNNL approach was to use radiation-transport modeling in conjunction with laboratory measurements to interpret the gamma spectrum for each measurement and determine the depth of contamination.

Before initiating the Fluor contract, PNNL performed a set of measurements using an HPGe detector, a

${ }^{137} \mathrm{Cs}$ check source, and aluminum filters to test the relationship between the "Peak-to-Valley" ratio and the thickness of intervening absorbers. Aluminum was used as a surrogate for concrete because its density is similar $\left(2.7 \mathrm{~g} / \mathrm{cm}^{3}\right.$ compared to $\left.2.35 \mathrm{~g} / \mathrm{cm}^{3}\right)$, and its atomic number is close to the atomic numbers of major concrete constituents. Ten-minute counts were taken for each of four configurations: an unshielded source, and three shields ranging from 0.16 to $0.64 \mathrm{~cm}$. For each configuration, a gamma spectrum was collected by the spectrometer, the count rate under the full-energy peak was determined, and a count rate for the region corresponding to multiple-scatter events (the "valley") was also determined. The resulting ratios for the full-energy count rate to the valley count rate are tabulated in Table 2.1.

Table 2.1. Ratios for Full-Energy to Valley Count Rates

\begin{tabular}{|c|c|}
\hline Configuration & Peak-to-Valley Ratio \\
\hline No shield & 10.31 \\
\hline $0.16 \mathrm{~cm} \mathrm{Al}$ & 10.00 \\
\hline $0.31 \mathrm{~cm} \mathrm{Al}$ & 9.85 \\
\hline $0.64 \mathrm{~cm} \mathrm{Al}$ & 8.64 \\
\hline
\end{tabular}

This test indicated that there is a change in the ratio as the thickness of the intervening material increases from 0 thickness through a thickness corresponding to a depth anticipated for radionuclide contamination in the basin walls. However, the difference in ratios was small enough that care had to be taken during the measurements to minimize the impact of interferences such as background radiation.

The detection of gammas emitted by basin wall and floor contamination is complicated by the emission of gammas by other material in the basin. There are three primary sources of this background radiation: 
spent fuel stored in racks on the floor, sludge on the floor, and ${ }^{137} \mathrm{Cs}$ dissolved in the basin water. The following considerations were made to minimize the effect of background radiation:

- Spent fuel: While a spent-fuel rod is an intense emitter of gamma radiation, water provides effective shielding against the radiation. When a fuel rod is positioned in air, an exposure rate of $380 \mathrm{R} / \mathrm{h}$ can be measured at $1 \mathrm{~cm}$ from the surface. However, underwater the exposure rate is considerably reduced. A calculation using the MCNP radiation transport code was performed which showed that the exposure rate falls to $1 \mathrm{R} / \mathrm{h}$ at 2 feet when the fuel rod is underwater, $0.08 \mathrm{R} / \mathrm{h}$ at 3 feet, $6 \mathrm{mR} / \mathrm{h}$ at 4 feet, and $0.03 \mathrm{mR} / \mathrm{h}$ at 6 feet. Thus, a 6 - $\mathrm{ft}$ layer of water between the detector and a fuel rod was determined to be sufficient to minimize the effect of spent-fuel background signals from interfering with the gamma spectrum collected while taking wall and floor measurements.

- Sludge: Sludge on the basin floor is capable of providing sufficient background to interfere with the floor measurements. Environmental concerns (agitation of sludge to the point that it results in an airborne release) limited cleaning of the basin floor to an area approximately the size of a single fuel canister. Every effort was taken to move floor sludge as far as possible from the measurement location. These efforts included 1) low-pressure washing with a trident-shaped wand in excess of 100 psig, 2) high-pressure washing with the trident-shaped wand at in-excess of $1000 \mathrm{psig}$, and 3) abrasive cleaning with a stainless-steel wire brush approximately the same diameter as the fuel canister. Each technique was applied multiple times in each measurement location.

- ${ }^{137} \mathrm{Cs}$ dissolved in the water: ${ }^{137} \mathrm{Cs}$ concentration in the water itself was sufficient to generate counts that would influence the signal from the concrete surface. Shielding calculations were performed to predict the count rate in the detector from the unshielded water, from the water with shielding around the detector, and from the wall contamination. These calculations were performed using the GammaRay Spectrum Synthsizer Code (SYNTH) and MCNP. Based on basin-water sampling, water configurations assumed that the water surrounding the detector contained a uniform $5 \mu \mathrm{Ci} / \mathrm{L}$ of ${ }^{137} \mathrm{Cs}$. The wall configuration assumed that the contamination was $12.9 \mu \mathrm{Ci} / \mathrm{cm}^{2}$, as found on the 1980 core samples (Bechtold 1981). With these assumptions, the detector count rates in Table 2.2 were estimated:

Table 2.2. Estimated Detector Count Rates

\begin{tabular}{|c|c|c|}
\hline $\begin{array}{c}\text { Radiation } \\
\text { Source }\end{array}$ & Shielding/Collimator & $\begin{array}{c}\text { Peak Count } \\
\text { Rate (c/s) }\end{array}$ \\
\hline Water & None & 62,860 \\
\hline Water & 2-in Pb shields & 2,389 \\
\hline Wall & 0.25 -in collimator & 359 \\
\hline Wall & 0.5 -in collimator & 1,433 \\
\hline Wall & 0.75 -in collimator & 3,235 \\
\hline
\end{tabular}


These results showed the importance of shielding the detector from the ${ }^{137} \mathrm{Cs}$ dissolved in the water. Without shielding, the data signal from the wall or floor would be overwhelmed by the gammas from the contaminated water. Calculations indicated surrounding the detector with the equivalent of 2 inches of lead shielding would reduce count rates due to the ${ }^{137} \mathrm{Cs}$ dissolved in the basin water to acceptable levels. 


\subsection{Equipment Selection and Development}

\subsection{Canberra High-Purity Germanium Detector}

An HPGe detector was selected to measure the gamma radiation emitted by wall surfaces. The germanium crystal operates at liquid nitrogen $\left(\mathrm{LN}_{2}\right)$ temperatures, so it is cooled by a cryostat that holds enough $\mathrm{LN}_{2}$ for several days, which equates to 3 to 4 days of operation without a refill. The detector used in this study was a Canberra model GC3518, with a "Big" multi-attitude cryostat (MAC) (Figure 3.1) (see Appendix A for detailed information). The detector has a closed-end coaxial geometry, is rated at $35 \%$ relative efficiency, and has a nominal resolution of $1.78 \mathrm{keV}$ FWHM at $1.33 \mathrm{MeV}$ and a peak/Compton ratio of 60.9:1. This HPGe detector was chosen for this task because it was the highest-resolution gamma spectrometer available. It is also capable of resolving peaks corresponding to gammas emitted by many different radionuclides. It was anticipated that ${ }^{137} \mathrm{Cs}$ would be the dominant gamma-emitting contaminant in the basin water and on the walls. However, if other gamma emitters were present, the HPGe instrument was capable of identifying them.

The HPGe was operated with associated electronics, including a preamplifier, amplifier, high-voltage power supply, analog digital converter (ADC), and multi-channel analyzer (MCA). The preamplifier is built into the detector housing; the other electronic modules were located externally (ultimately positioned above the basin grating) and were connected to the detector by cabling.

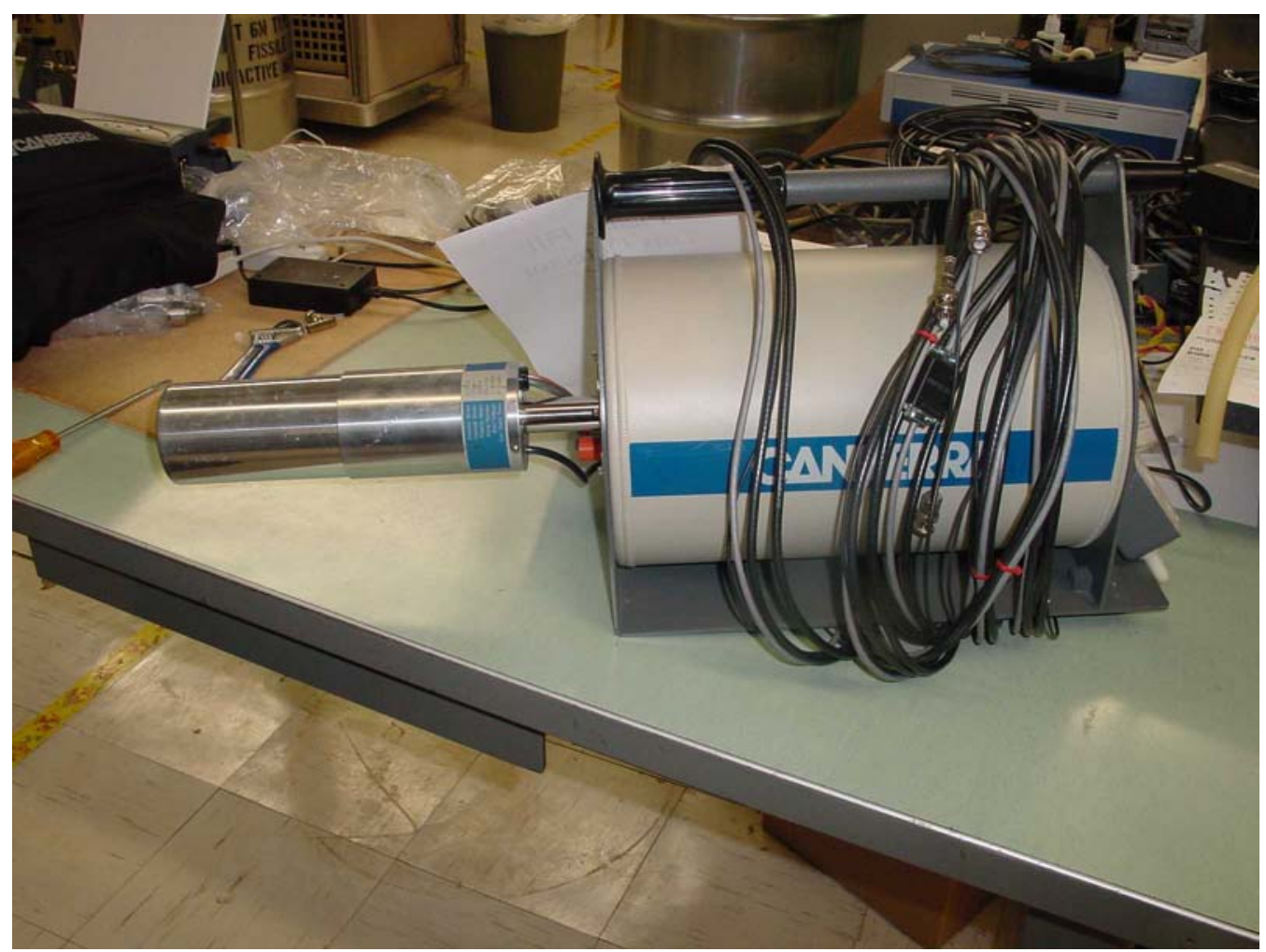

Figure 3.1. Canberra Model GC3518 Detector with a "Big MAC” Cryostat 
The HPGe detector was housed in a submersible vessel that enabled underwater operation. ANTEC Technology provided the submersible vessel (see Figure 3.2) (see Appendix B for the detailed sketch). The vessel was constructed of stainless steel and was designed to include the HPGe detector, lead shielding (also provided by ANTEC), a tungsten collimator, and $\mathrm{LN}_{2}$ cryostat. The HPGe detector system had a total weight of approximately 500 pounds. The power supply and data-acquisition cables were fed through watertight connections in the vessel. The submersible vessel included several important features worth mentioning, namely:

- The capability to refill the detector cryostat with $\mathrm{LN}_{2}$ without opening the vessel. It was anticipated that the cryostat would require filling approximately every three days during basin operations.

Although it is possible to keep the detector system submerged with an empty cryostat, the cryostat must be charged (ensuring that the germanium crystal remains sufficiently cool) before measurements could be taken. The vessel also included a vent tube of sufficient length to vent the nitrogen gas above the basin grating.

- Standoff bolts to ensure that the face of the detector would not touch the basin wall, reducing the potential for the detector system window to become contaminated. The standoff bolt also provided a consistent geometry (including the water) between the detector and the wall.

- A removable faceplate providing the ability to switch out collimators without removing the entire detector from the submersible vessel. Three collimators were fabricated for taking wall measurements. The first collimator was a tungsten plug for use in establishing a baseline background count rate. The other two tungsten collimators had been precision drilled with centered boreholes of $3 / 8$ and $3 / 4$ inches respectively, providing collimator flexibility, depending on actual count rates experienced in the basin.

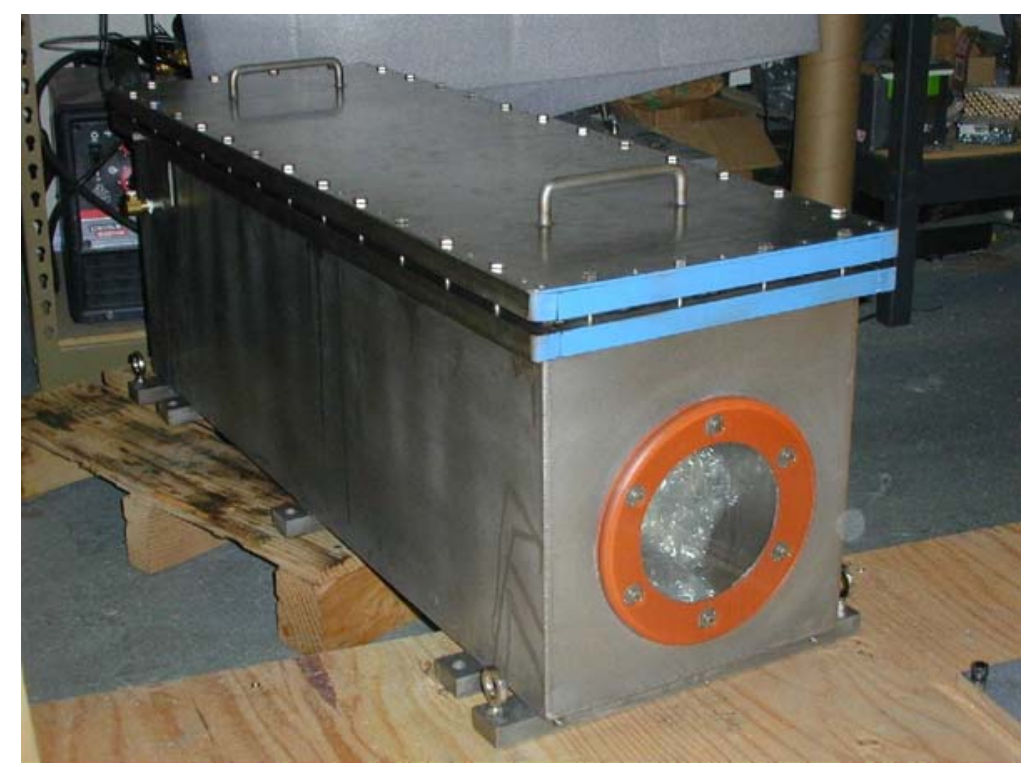

Figure 3.2. ANTEC Submersible Vessel Upon Delivery 


\subsubsection{Calibration of the HPGe Detector}

The HPGe detector system was calibrated using a radioactive source containing $2.03 \mu \mathrm{Ci}$ of ${ }^{137} \mathrm{Cs}$. A series of concrete attenuators were used to simulate radioactive penetration into concrete. As was mentioned previously, the HPGe detector system included three tungsten collimator configurations: a tungsten plug for performing a background calibration once in the basin, a $3 / 8$-inch collimator, and a 3/4-inch collimator. Calibrations were performed using the above-mentioned source with the following: no collimation, $3 / 8$-inch collimator, and $3 / 4$-inch collimator. Calibrations were performed using different thicknesses of concrete attenuators to simulate radioactive penetration.

Included in Attachment A is a detailed sketch of the HPGe detector. Using a simplified version of this system, including the submersible vessel, an MCNP model was constructed. The MCNP model broke up the simplified sketch into material types, surfaces, and dimensions. MCNP simulations involved the use of the $3 / 8$ - and $3 / 4$-inch collimators, the $2.03 \mu \mathrm{Ci}$ of ${ }^{137} \mathrm{Cs}$ source, and various attenuator thicknesses.

Measured values versus simulated (or calculated) values for the HPGe detector system in the submersible vessel with the $3 / 4$-inch collimator, detector in air, are found in Table 3.1. The following data set is included to document the process used and to provide a point of reference for the $\mathrm{HgI}_{2}$ detector-system calibrations discussed later in the document. This data set proved to be, after analysis of the spectrum, the most representative of the ${ }^{137} \mathrm{Cs}$ source and the ultimate deployment configuration.

Table 3.1. HPGe Detector in Submersible Vessel in Air with $3 / 4$-inch Tungsten Collimator in Front of Detector (2.03 $\mu \mathrm{Ci}$ large-area ${ }^{137} \mathrm{Cs}$ source)

\begin{tabular}{|c|c|c|c|c|c|}
\hline \multirow{2}{*}{$\begin{array}{l}\text { Attenuator } \\
\text { Thickness } \\
(\mathbf{m m})\end{array}$} & \multicolumn{3}{|c|}{ Count Rate (counts/second) } & \multicolumn{2}{|c|}{ Peak-to-Valley Ratio } \\
\hline & $\begin{array}{c}\text { Part of } \\
\text { Spectrum }\end{array}$ & Measured & Calculated & Measured & Calculated \\
\hline \multirow{2}{*}{0} & Valley & 5.99 & 3.84 & 4.63 & 4.58 \\
\hline & Peak & 27.8 & 17.6 & \multicolumn{2}{|c|}{$1.0 \%$ difference } \\
\hline \multirow{2}{*}{2} & Valley & 5.79 & 3.81 & 4.54 & 4.45 \\
\hline & Peak & 26.3 & 17.0 & \multicolumn{2}{|c|}{$2.0 \%$ difference } \\
\hline \multirow{2}{*}{5} & Valley & 5.60 & 3.68 & 4.51 & 4.33 \\
\hline & Peak & 25.3 & 15.9 & \multicolumn{2}{|c|}{$4.2 \%$ difference } \\
\hline \multirow{2}{*}{10} & Valley & 4.87 & 3.46 & 4.32 & 4.21 \\
\hline & Peak & 21.1 & 14.5 & \multicolumn{2}{|c|}{$2.8 \%$ difference } \\
\hline \multicolumn{6}{|c|}{$\begin{array}{l}\text { Note: The }{ }^{137} \mathrm{Cs} \text { source used for this experiment had a non-homogeneous distribution, so the } \\
\text { modeled case assumed a source strength different than that observed in the } \\
\text { measurement. This caused the measured counts per second to be different from the } \\
\text { modeled rate, but the peak-to-valley ratios were comparable. }\end{array}$} \\
\hline
\end{tabular}

\subsubsection{Deployment Platform for the HPGe Detector}

A system was designed and fabricated to move and position the HPGe detector at specific basin locations for data collection. K East Basin Operations management and Nuclear Chemical Operators were involved throughout the design and integration phases to minimize and address operational concerns. The deployment platform for the HPGe detector system had the following attributes: 
- Enabled the system to hang below the 11/2-in. grating-transfer slot. The system was moved using a basin overhead hoist.

- Designed for the detector system and its associated cabling to remain submerged throughout the deployment with the exception of refilling the cryostat with $\mathrm{LN}_{2}$.

- The deployment platform remained stationary while the detector vessel moved vertically. The deployment platform was designed for the detector vessel to take measurements from 1 foot below the basin water line to a depth of 9 feet below the water line.

- Designed to move the detector system horizontally, enabling the detector standoffs to touch the basin wall as measurements were taken and move away from the wall as the detector was moved to depth. This motion was accomplished with mechanical flexible shafts, worm gears, and a rack-and-pinion system.

- All load-bearing aspects of the deployment platform were load tested and tagged.

\subsubsection{HPGe Detector System Operational Testing}

The HPGe detector system and its associated deployment platform were operationally tested in the Fluor Hanford 306 East facility. Scaffolding (Figure 3.3) was used to suspend the detector and deployment system. Operational testing began with checking the movement of the detector both vertically and horizontally. The vertical movement was needed to raise and lower the detector to depth. The vertical movement functioned as planned. The horizontal movement was needed to move the detector toward and away from the basin wall. The large cantilevered load generated by the shielded and collimated detector system proved problematic for the horizontal movement subsystem. When extended towards the wall, the offset load was difficult to retract and caused the deployment system to cant forward. Displacement support legs were added to the deployment platform to minimize the cant, resulting in the horizontal movement performing as expected. 

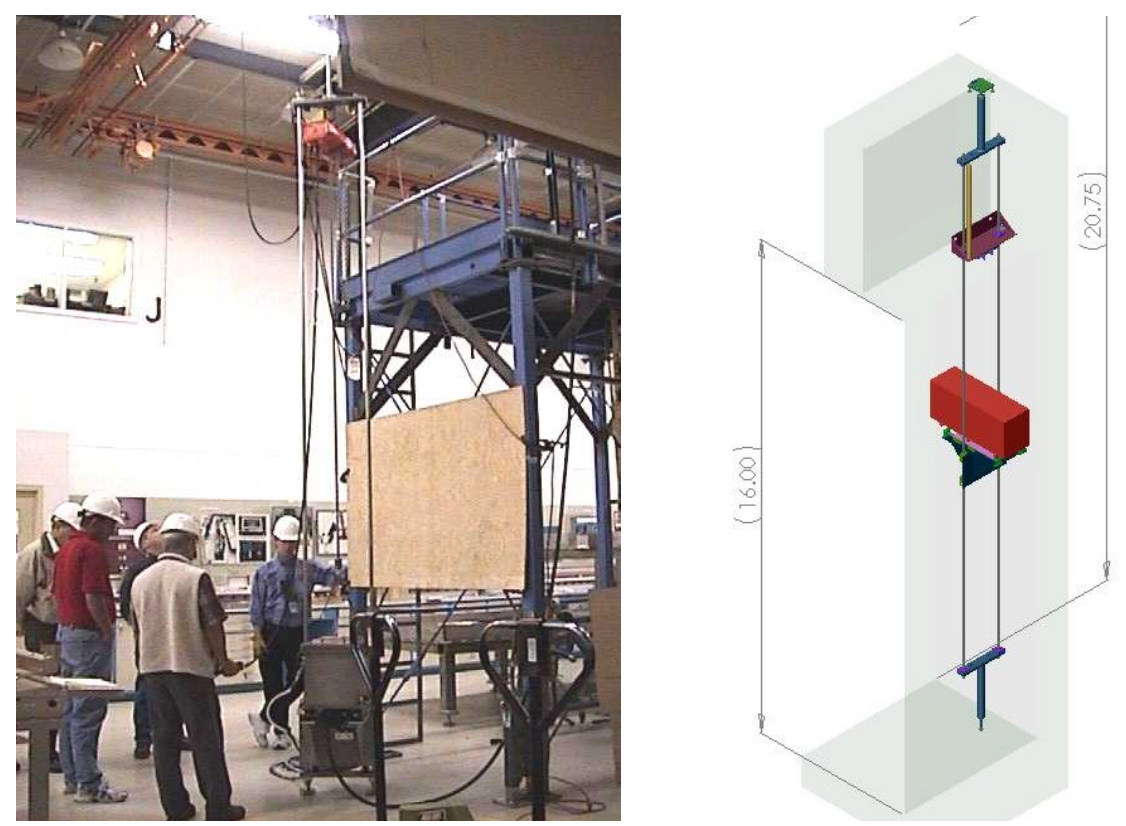

Figure 3.3. HPGe Detector System Testing in 306 East

A large (140 gal) tank was used to leak-test the detector system and to perform the underwater calibration of the detector. Additionally, the test bed in 306 East was used to gain experience with collimator change-outs and $\mathrm{LN}_{2}$ refill operations.

K East Basin Operations management and Nuclear Chemical Operators observed the detector and deployment system operation and identified no significant hazards. K Basin planning personnel used the 306 East operational testing to complete the work steps described in the work package.

\subsubsection{HPGe Detector System Deployment}

The HPGe Detector system was shipped to the K East Maintenance Building on May 21, 2002, 14 weeks from project initiation. The system was deployed on July 2, 2002. The delay in deployment was due primarily to performing the necessary analyses and evaluations to complete and approve the work package.

On July 3, 2002, three background measurements were taken in the center bay (approximately 10 feet from the edge of the opening in the grating of the Center Bay) at depths of 3 feet, 6 feet, and 9 feet below the water surface. Due to the July 4 holiday, the continuation of the deployment was delayed until July 8 , 2002. Operational support was provided the evening of July 9, 2002, at which time water was found in the submersible vessel. Aggressive pressure washing, including nozzle contact, of the surface of the submersible vessel (not originally expected) may have contributed to the leak. Another possibility for the leak is that the rapid drop in temperature from a warm outside location $\left(28^{\circ} \mathrm{C}\right)$ into the cool basin water $\left(13^{\circ} \mathrm{C}\right)$ may have resulted in some steel shrinkage, loosening the lid seal on the submersible vessel. However, the exact cause of the leak is still unknown.

Several attempts were made to recover and decontaminate the submersible vessel and detector. Ultimately, it was not possible to repair the HPGe detector system due to two primary factors: 1) the risk of radioactive exposure to staff involved in the decontamination and repair of the system and 2) the impact of the repair activity on ongoing basin operations. Recent experience with the removal of other 
equipment from the basin indicated that the HPGe detector system was very likely to be sufficiently contaminated to cause an airborne contamination concern. The airborne contamination would place the repair team, which was to include both Fluor and PNNL staff, at greater risk of exposure. Preventive measures to reduce the airborne-contamination risk would result in curtailing all other basin activity, including high-priority fuel removal and sludge-retrieval readiness activities. The decision not to repair the HPGe detector system placed additional importance on attempting at least one wall measurement using the floor detector system.

\subsection{Constellation Mercuric-Iodide Detector}

Although the HPGe detector described above was the highest-resolution gamma spectrometer available and was deemed capable of providing depth of contamination, the physical size of the HPGe detector system made it unsuitable for obtaining measurements from the basin floor. The HPGe detector system in its submersible vessel was too large to fit through the spent-fuel storage racks, making it impossible for the detector system to contact the floor. Additionally, the shielding designed for the HPGe detector system was determined to be insufficient to shield against the higher radiation dose expected on the floor due to the proximity of spent fuel and sludge. These limitations made it necessary to identify an alternative approach to taking floor measurements. An additional benefit associated with introducing another detector system was the ability to take wall and floor measurements at the same time, which was important given the original aggressive schedule for taking measurements.

A mercuric-iodide $\left(\mathrm{HgI}_{2}\right)$ detector manufactured by Constellation Technology Corporation was selected for taking floor measurements (see Appendix C). The selected $\mathrm{HgI}_{2}$ detector was quite compact, capable of fitting in a human hand (Figure 3.4). The $\mathrm{HgI}_{2}$ detector specified an electronic-pulse shaping time of up to $24 \mu$ s (the time required for the electronics to completely collect a charge from the gamma radiation deposited on the detector crystal). The $24-\mu$ s setting is the time required for optimum performance at the $662-\mathrm{keV}$ energy of interest for this application. Ionization from gamma-ray interaction within the active volume of the detector generates electron-hole pairs that are collected by high voltage applied to either side of the detector crystal. The electrons are very mobile and are quickly collected. However, the holes

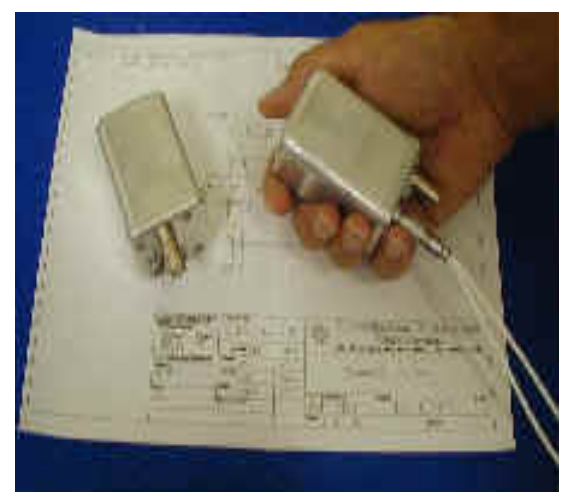

Figure 3.4. Constellation Technology Mercury-Detector Module

limit the speed at which complete charge is collected, determining the ultimate data-collection rate for the system. For a $25-\mu$ s shaping time, the top collection rate is 40,000 counts per second.

Hardware was identified that would handle the required 20 to $25-\mu$ s shaping time generated by the detector in response to gamma-ray energy depositions. A Tennelec 242 nuclear instrumentation module (NIM)-based amplifier was used with a recently developed AMPTEK 8000A MCA, which stored the 
processed gamma-ray pulses as a spectrum. Signals from the detector were processed through an NIMbased ADC capable of processing the $25-\mu$ s pulse-height signals.

An off-the-shelf stainless-steel vacuum fitting (8.0-in. O.D. flanges) was chosen as the submersible vessel, able to contain the detector and the size and weight of the tungsten shielding (Figure 3.5 and Figure 3.6). A sketch of the $\mathrm{HgI}_{2}$ submersible vessel can be found in Appendix D. Standard copper gaskets were used to ensure a watertight seal. A set of three existing tungsten annulus shields was modified to contain the $\mathrm{HgI}_{2}$ detector. The modification resulted in a tungsten wall thickness of $3.4 \mathrm{~cm}$. Signal and low-voltage power cables were fed through a hole in the wall of the middle tungsten annulus to allow external electronics a connection to the internal circuitry. A 5-cm-thick lead collimator with a $0.635-\mathrm{cm}$-diameter hole limited the field of view of the floor to roughly $0.317 \mathrm{~cm}^{2}$. The vacuum flange endplate was also machined to minimize shielding from the stainless steel while maintaining a watertight chamber. The steel-viewing window was approximately $2 \mathrm{~mm}$ thick.

The Tennelec 242 NIM-based amplifier supplied power to the detector using a standard 9-pin preamp cable fed to the detector through a section of thick-walled Tygon Flexible Tubing. The properties of Tygon Flexible Tubing were determined to be acceptable in the radiation fields expected for this activity. (See e-mail in Appendix D from William J. Knight from the "As Low as Reasonably Achievable" (ALARA) Center - "....reference literature states that flexible PVC-based products will begin to show a $25 \%$ loss of elongation [hardening or becoming brittle] after 20 Mrads exposure.") The Tygon Flexible Tubing was judged to be sufficiently flexible and coiled such that little radiation streaming was expected from sludge or fuel while the detector system was on the basin floor.

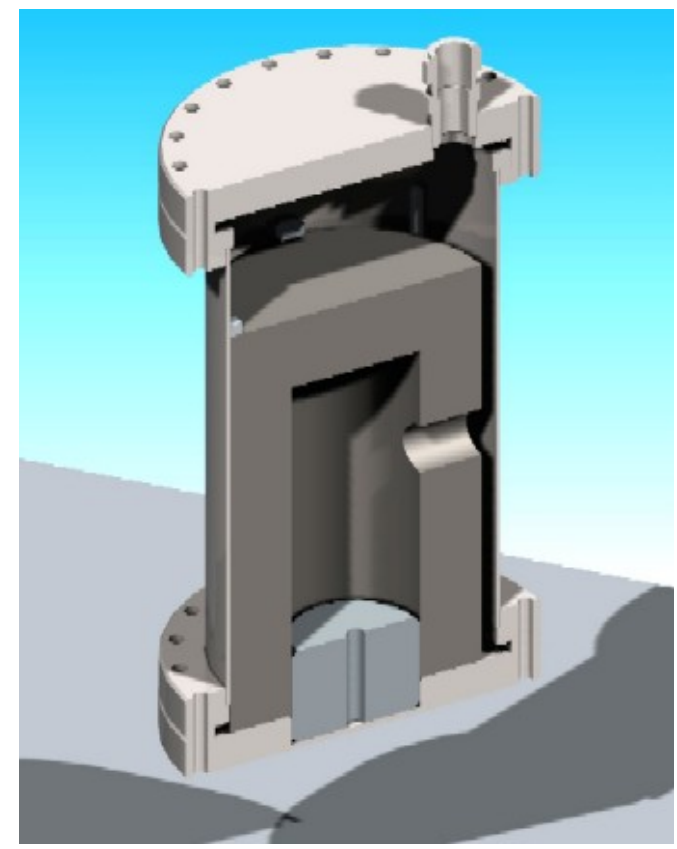

Figure 3.5. Cut Away Drawing of the $\mathbf{H g I}_{2}$ Submersible Vessel 


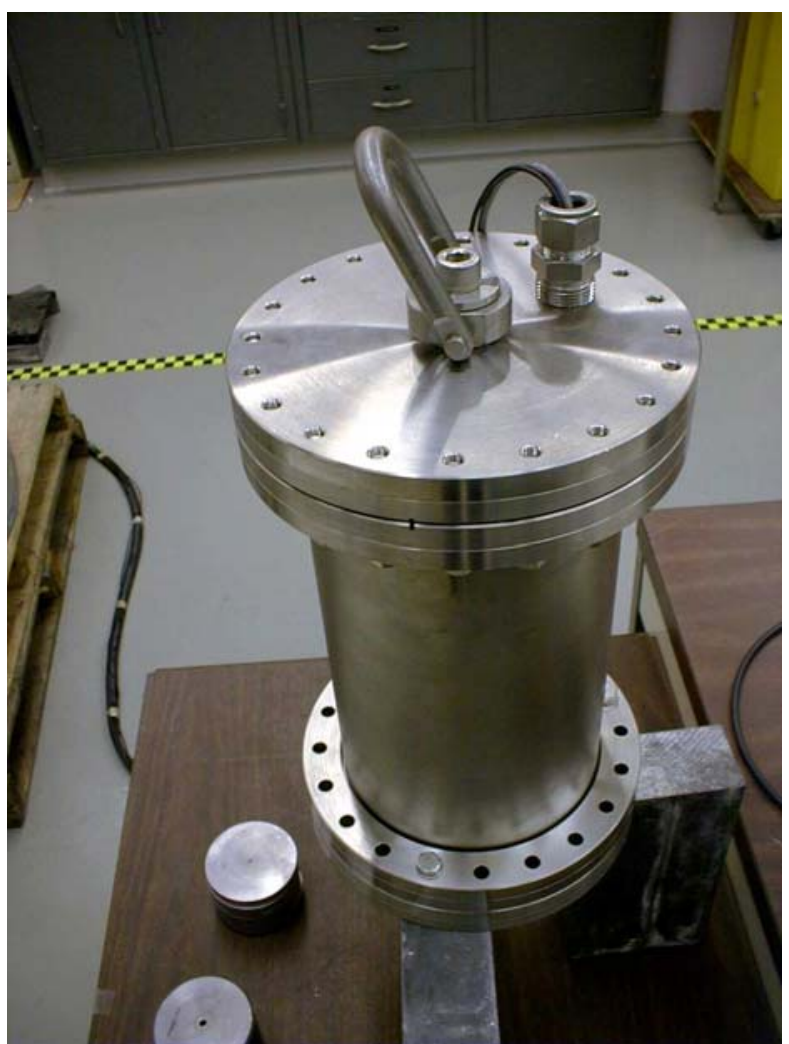

Figure 3.6. HgI $_{2}$ Detector System Submersible Vessel

As mentioned previously, the chief benefit of the $\mathrm{HgI}_{2}$ detector system, which included the detector, tungsten shielding, lead collimator, and the submersible vessel, was its compact size, making it possible to take floor measurements with the spent-fuel canister racks in place. Another benefit was that the $\mathrm{HgI}_{2}$ detector system did not contain an $\mathrm{LN}_{2}$ cryostat or have a requirement to be cooled, significantly reducing the deployment complexity. With the compact size of the detector system and the availability of tungsten for shielding, the detector system was ready for deployment within the required time constraints. The $\mathrm{HgI}_{2}$ detector system was designed as a compromise between detector size, detector resolution, shielding requirements, and room-temperature operation. It was envisioned that the $\mathrm{HgI}_{2}$ detector would be put into the basin and moved periodically as targeted basin floor locations became available and removed from the basin after wall measurements were completed.

The $\mathrm{HgI}_{2}$ detector system readiness was checked several ways. The water-tightness of the system was determined by lowering the $\mathrm{HgI}_{2}$ detector system into a large-diameter $\mathrm{PVC}$ pipe located in the well of the 329 Building. The pipe was filled with a 20 -ft-high column of tap water. A $1-\mu \mathrm{Ci}^{137} \mathrm{Cs}$ source was attached to the underside of the $\mathrm{HgI}_{2}$ detector system and counted overnight. The $\mathrm{HgI}_{2}$ detector system was then retrieved and opened to check for water ingress around the copper seals. No water ingress was found, which affirmed water-tightness.

A new copper gasket was attached, and the $\mathrm{HgI}_{2}$ detector system was again sealed. The system was then subjected to a 2400-psig high-pressure water treatment for 5 minutes to assure that the system was watertight under a thorough cleaning scenario. The Tygon tubing, marine sealant, and gaskets all held up to the high-pressure water treatment without any signs of water leakage. This second test was performed after the lessons learned from the deployment of HPGe detector system. 
After a new copper gasket was installed and the $\mathrm{HgI}_{2}$ detector system was again sealed, the decision was made to deploy the system.

\subsubsection{Mercuric-Iodide-Detector Deployment Platform}

Deployment of the $\mathrm{HgI}_{2}$ detector was straightforward and did not require an engineered deployment platform. The stainless-steel vacuum flange endplate that was the top of the submersible vessel included a lifting bail. A wire cable was attached to the lifting bail. The wire cable was tied off to a basin hoist when the floor detector system was moved from one location to another. Once the detector was positioned over the desired location, the hoist was lowered until the detector was positioned on the basin floor.

\subsubsection{Mercuric-Iodide-Detector Deployment}

The original plan was to deploy the $\mathrm{HgI}_{2}$ and HPGe detector systems at the same time. It seemed conceivable to do so because it was assumed that the $\mathrm{HgI}_{2}$ detector system would be relatively easy to deploy in comparison to the HPGe detector system. However, it became very obvious that deployment of either system was going to be a challenge. Given that schedule (completing the deployment by May 2002) was the primary driver in approach, the more complicated and resource-intensive HPGe detector system was given priority over the $\mathrm{HgI}_{2}$ detector system. Once it became known that the HPGe submersible vessel had leaked, considerable effort went into planning for the recovery and re-deployment of the system. In December 2002, due primarily to a lack of progress in recovering and deploying the $\mathrm{HPGe}$ detector system, primacy was placed on the $\mathrm{HgI}_{2}$ detector system deployment.

When the project was initiated, it was assumed that an existing Tri Nuclear pump would be used to remove sludge from the basin floor before taking floor measurements. However, as the $\mathrm{HgI}_{2}$-detectorsystem deployment approached, it was discovered that the Tri Nuclear pump was not available. A new method for sludge removal had to be developed. Several options were considered and evaluated against basin requirements. The approach selected was to fabricate a device similar to a fuel canister coupled with a spray wand (Figure 3.7). One side of the canister was slotted to allow for sludge removal from the desired location. The canister was also designed to support insertion of the $\mathrm{HgI}_{2}$ detector once the area

had been cleaned. The other side of the canister had a steel bar welded across it to prevent insertion of the $\mathrm{HgI}_{2}$ detector into the unwashed location. The spray wand was designed to attach to the KE Basin demineralized water system ( $\sim 110$ to $150 \mathrm{psig})$ or a stand-alone pressure-wash system ( $1200 \mathrm{psig})$. The standoff distance of the nozzles was of particular concern. An analysis of the nozzle performance indicated a very sharp decline in water velocity over relatively short distances. [The Submerged Fan Jet analysis has been included in the data package provided to FH (R.B. Baker).] The spray and/or wash approach was to minimize the standoff distance and yet remain within the KE Basin operational envelope.

Agreement was reached with K East Basin Operations management and Nuclear Chemical Operators to hold the standoff distance to approximately $1 \mathrm{inch}$. A mechanical stop was used to maintain the standoff distance.

Testing was conducted in 306 East Building to determine the effectiveness of the sludge-removal system. Sludge simulant from previous SNF work was used in the effectiveness testing (Schmidt and Elmore 2002). Figure 3.8 illustrates that at $\sim 90$ psig, the sludge simulant was mobilized and removed from the slotted canister. K East Basin Operations management and Nuclear Chemical Operators were involved in the $\mathrm{HgI}_{2}$ deployment planning, including the engineering and operation of the sludge-removal system. To expedite the $\mathrm{HgI}_{2}$ detector system deployment, the work package (work steps, ALARA Plan, hazards 
analysis, etc.) was prepared during the sludge-removal testing. Once the operational requirements had been met and the $\mathrm{HgI}_{2}$ detector system, including the sludge removal system, had competed functional testing, preparations were made to initiate deployment of the system.

The $\mathrm{HgI}_{2}$ detector system was deployed into the K East Basin on January 27, 2003. The first cubicle location, as well as all remaining locations, was based on the recommendation of the SNF Transition Projects staff. A standard operating procedure was developed for taking floor measurements, including the work steps necessary for each measurement. A log sheet was completed for each measurement location, indicating the water pressure and number of $180^{\circ}$ rotations of the spray wand used to remove sludge. If a single location received additional cleaning, a separate log sheet was completed to record the activity. Basin cameras and lights were used to visually inspect the location and assist in determining whether to perform additional cleaning before taking a measurement. A video was recorded of each location during each washing and/or cleaning cycle and after each wash and/or cleaning (Figure 3.9). A complete set of videotapes has been provided to the SNF Transition Projects.
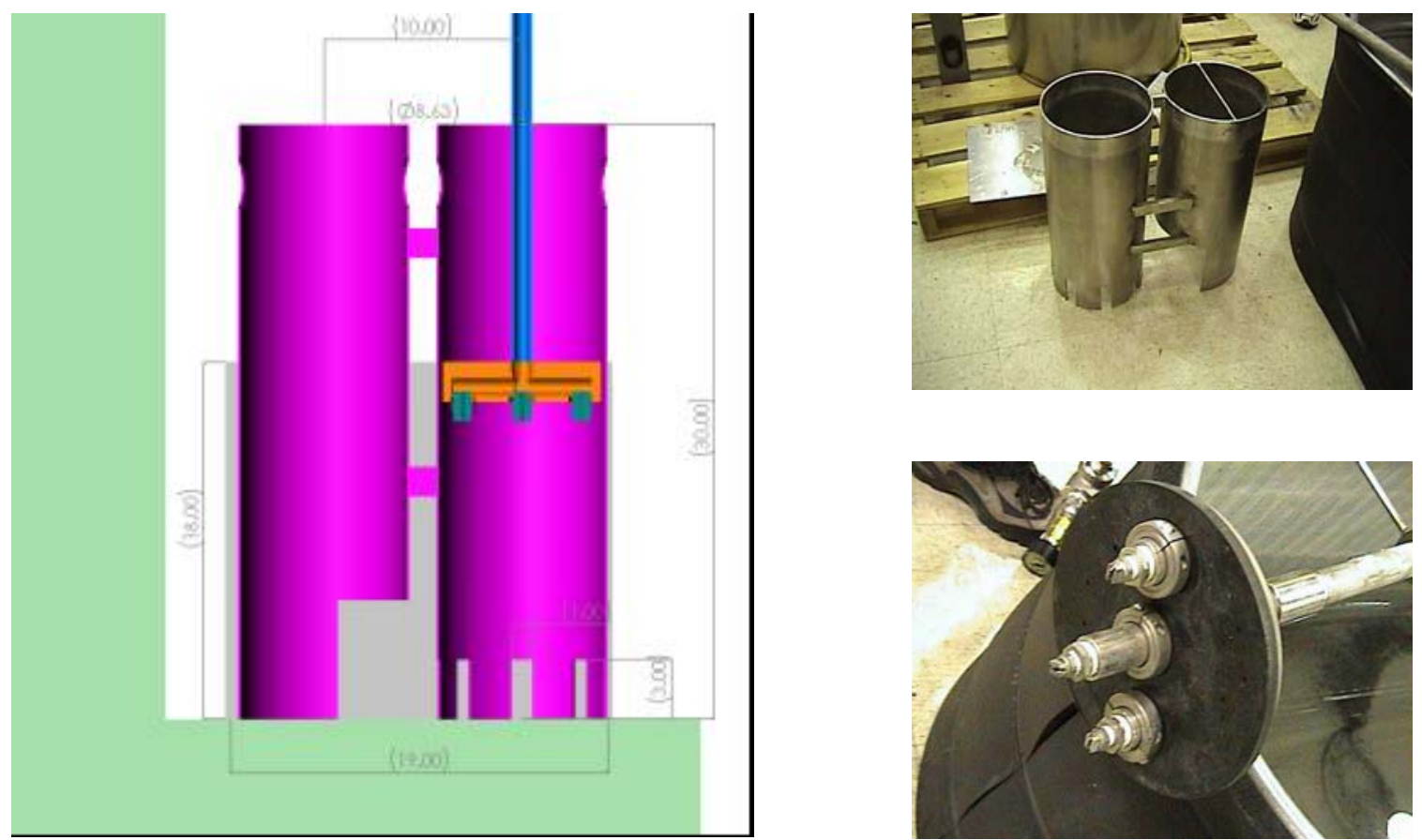

Figure 3.7. Sludge Removal—Slotted Canister Set and Spray Wand 


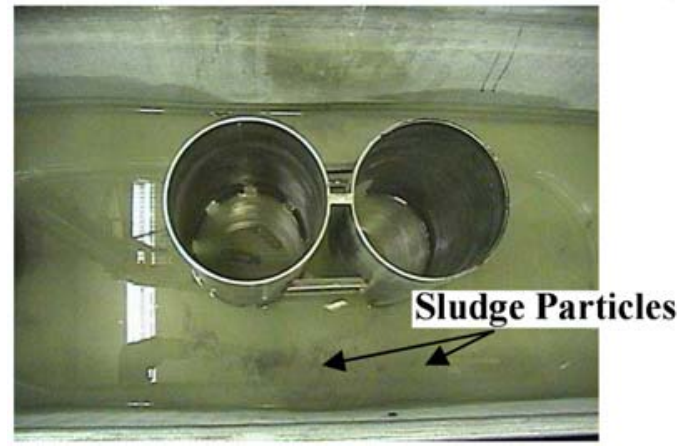

Figure 3.8. Sludge-Removal Effectiveness Testing in 306 East

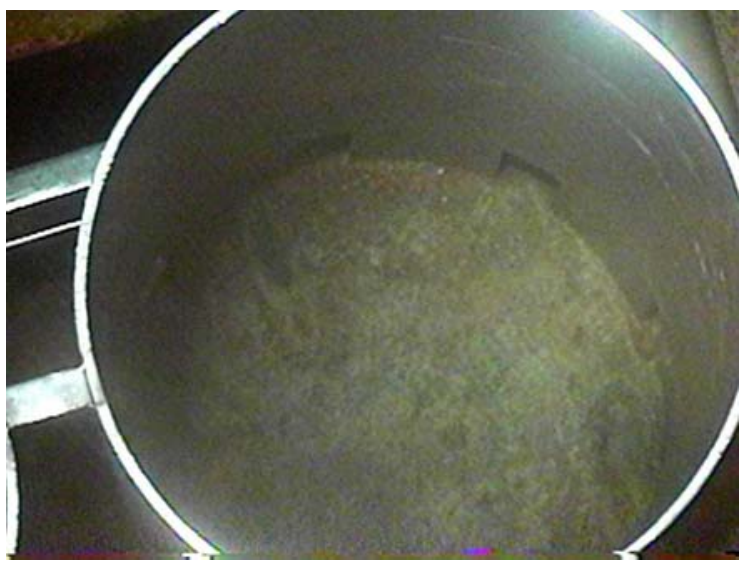

Figure 3.9. Video Picture of Cubicle 2209 West After 110 psig Wash

The detector was not inserted into the canister until the floor appeared to be clean (based on visual examination), and the haze cloud had settled. A short 3- to 5-minute reading was taken (and the spectra captured) to ensure that the detector was operating properly. Each measurement was run for approximately 20 to 24 hours, ensuring sufficient data to analyze. Amplified signals from the detector were digitized and stored in a multi-channel analyzer. The resulting spectra were then transferred to a laptop computer for data storage and readout. The data were transferred from the laptop computer via $3 \frac{1}{2}$-in. floppy diskettes. A JPG picture of the spectra was saved along with the data files.

Once the $\mathrm{HgI}_{2}$ detector system was deployed, the level of radionuclide activity was found to be much higher than anticipated. As a result, in consultation with the SNF and Transition Projects staff, a stainless-steel brush was designed and fabricated as an attempt to reduce the radionuclide activity level by loosening and removing sludge particles that remained after pressure washing (Figure 3.10). In general, brushing the floor did little to reduce the level of radionuclide activity. 


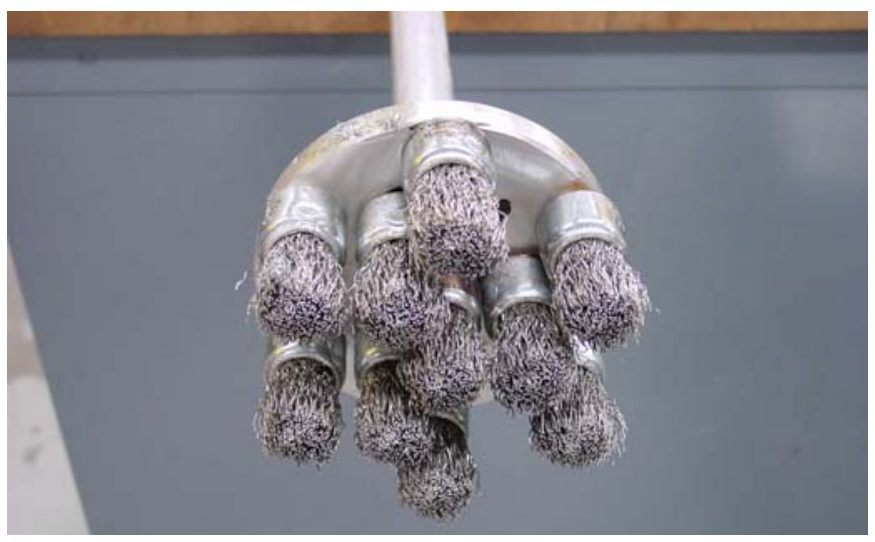

Figure 3.10. Stainless Steel Brush for Basin Floor

Based on the uncertainty regarding the recovery of the HPGe detector system, PNNL proposed using the $\mathrm{HgI}_{2}$ detector system to take at least one measurement from the basin wall. The proposed approach was to place the $\mathrm{HgI}_{2}$ detector system in a horizontal orientation by using a specially designed stainless-steel table. SNF Transition Projects approved the design and fabrication of a table (Figure 3.11). The table centered the $\mathrm{HgI}_{2}$ detector system 28.5 inches above the basin floor (Figure 3.12).

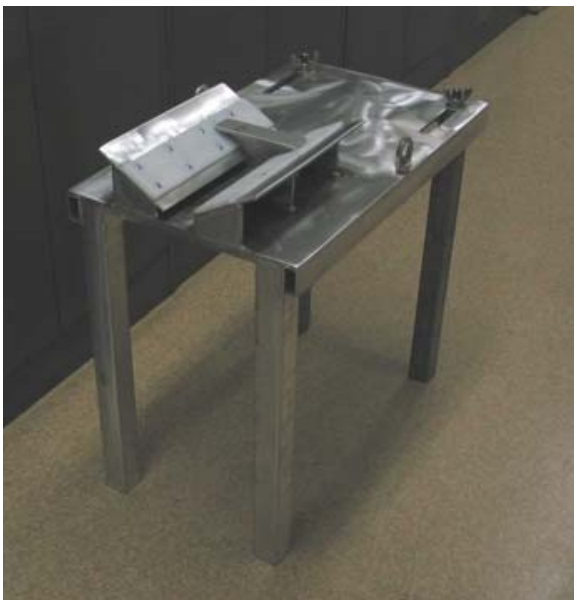

Figure 3.11. Table Designed to Take Wall Measurement Using $\mathrm{HgI}_{2}$ Detector System

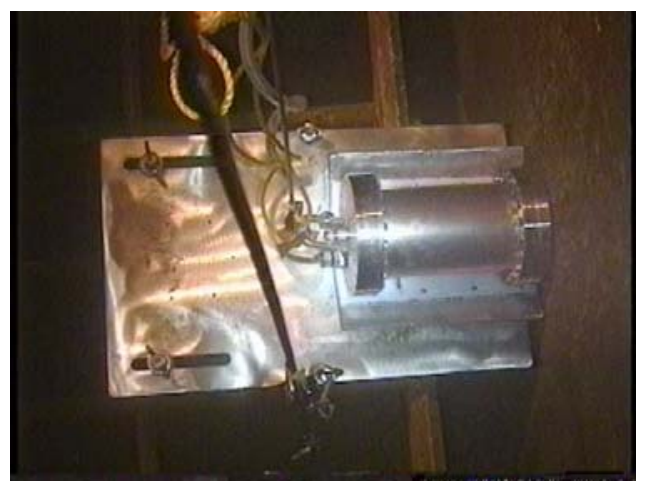

Figure 3.12. HgI $_{2}$ Detector System Taking Wall Measurement 
The table and $\mathrm{HgI}_{2}$ detector system were deployed into the East Bay of $\mathrm{K}$ East Basin to take a wall measurement. As was the case with the contamination levels found on the floor, the wall radionuclide contamination level was higher than originally expected. A stainless-steel brush, similar to the brush used on the basin floor, was designed and fabricated (Figure 3.13) to attempt contamination reduction through brushing. The wall brush, although more effective in reducing wall contamination than the floor brush was in reducing floor contamination, did not appreciably reduce the activity level on the wall.

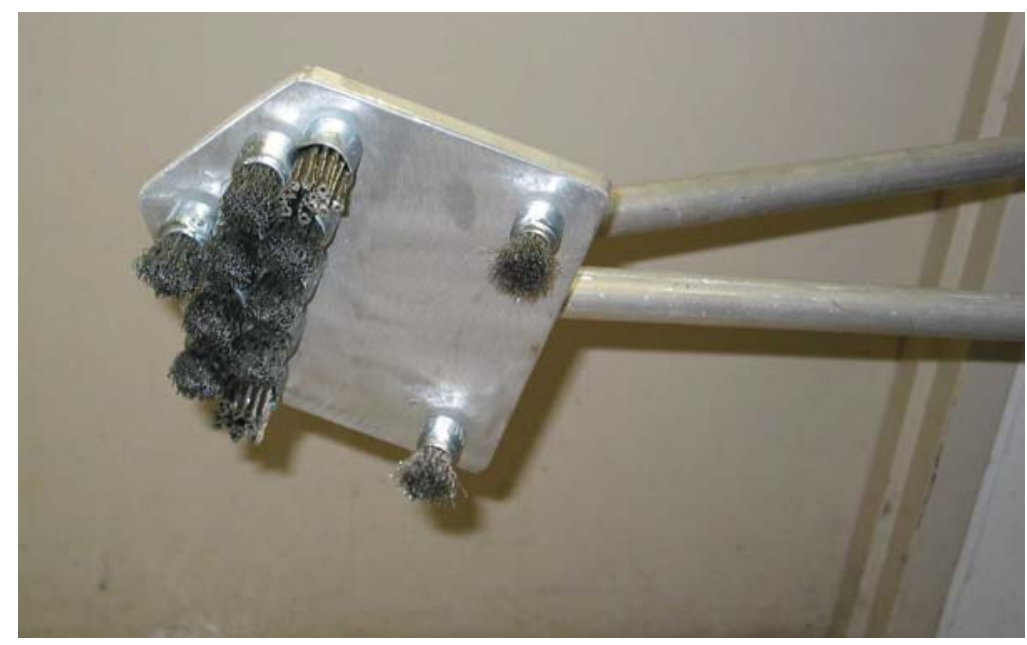

Figure 3.13. Stainless-Steel Wall Brush

On May 28, 2003, after completing all agreed to floor and wall measurements, a ${ }^{137} \mathrm{Cs}$ source was placed into KE Basin as a confirmatory calibration check of the $\mathrm{HgI}_{2}$ detector. The ${ }^{137} \mathrm{Cs}$ source was placed on the back side of a stainless-steel plate (Figure 3.14). The stainless-steel plate was specifically designed to center the detector over the ${ }^{137} \mathrm{Cs}$ source. The source plate was placed on the stainless-steel table used to take wall measurements. A $4 \frac{1}{2}$-inch PVC standoff was attached to the stainless-steel plate to minimize the effect of sludge that may have settled on the stainless-steel table. A 1-hour count was taken with the detector on the source plate. Another 1-hour count was taken at the same location (including the same height) without the source plate as a baseline count. 


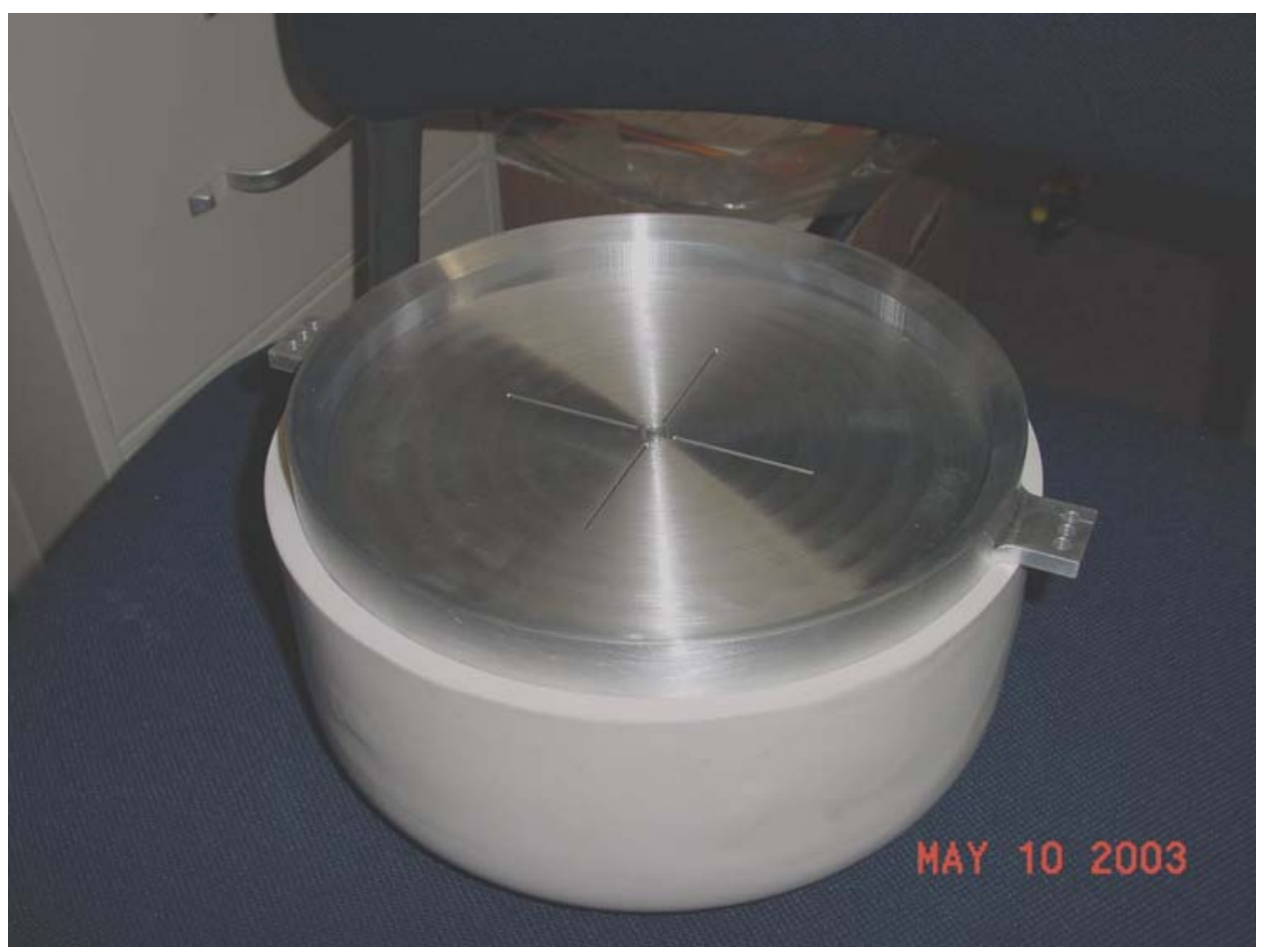

Figure 3.14. Calibration-Test Cesium Source

The $\mathrm{HgI}_{2}$ detector system performed well over the three months of intermittent operation in the basin. No changes in the detection characteristics were observed, supported by three measurements of a single location (cubicle 5209 east) yielding similar count rates and activity levels.

\subsubsection{Calibration of the Mercuric-Iodide Detector}

Energy calibration consisted of using a series of radiation sources with various discrete-energy gamma rays to determine the collection efficiency (expressed as counts per gamma ray) versus the particular gamma-ray energy. The resolution (width) of the corresponding spectral peak, expressed as the fullwidth at half-maximum (FWHM) value was also determined as part of the calibration.

Because of low hole mobility in $\mathrm{HgI}_{2}$, the chance of incomplete charge collection increases for this detector crystal relative to other types of semiconductor materials. The net result of incomplete charge collection is a lopsided peak that has the expected Gaussian shape on the high-energy side but a significant tail on the low-energy side. The measured FWHM folds the intrinsic width together with the added width due to incomplete charge collection, thus adding counts to the low-energy side of the spectral peak, which is also known as the "valley." For this campaign, the overall effect of low hole mobility is a greater uncertainty in the peak-to-valley ratio (hence, a greater uncertainty in the penetration depth of ${ }^{137} \mathrm{Cs}$ into the concrete walls) as compared to a detector material with high hole mobility, such as HPGe.

\subsubsection{Penetration Calibration Using Mercuric-Iodide Detector}

The $\mathrm{HgI}_{2}$ detector system was calibrated using a radioactive source containing $10 \mu \mathrm{Ci}$ of ${ }^{137} \mathrm{Cs}$. The same series of concrete attenuators that were used with the HPGe detector was also used with the $\mathrm{HgI}_{2}$ detector system to simulate radioactive penetration into concrete. Four different concrete attenuators $(2.5 \mathrm{~mm}, 5.0$ 
$\mathrm{mm}, 10.0 \mathrm{~mm}$, and $25.0 \mathrm{~mm}$ thick) were used for measurements corresponding to a source at several depths up to $42.5 \mathrm{~mm}$ of concrete.

Typical measurements involved counting the source at a fixed location and then placing a given thickness of concrete absorber between the source and the $\mathrm{HgI}_{2}$ detector. The net spectrum provides the effect of the given thickness of concrete on the detector response. Combinations of concrete standards allowed measurements to be obtained typically in 2.5- $\mathrm{mm}$ intervals. The maximum depth measured corresponded to a 42.5-mm thickness, as illustrated in Figure 3.15. The $\mathrm{HgI}_{2}$ detector has features similar to other solid-state detectors (such as HPGe), and several of the prominent features are identified in Figure 3.15.

Figure 3.15 clearly illustrates a marked drop in the number of gamma rays that deposit the full initial energy and fill the "Full-Energy Peak" region of the spectrum when compared to the number that fill the "Valley" region located slightly lower in energy. Also visible is the large increase in low-energy gamma rays that fill the region below the lead-x-ray region.

The lead $\mathrm{x}$ rays arise from x-ray fluorescence as gamma rays entering the collimator hole of the shielding excite the lead itself. The backscatter peak is caused by incoming gamma rays that initially interact with the detector shielding material; a lower energy gamma ray that is produced in the interaction is then detected by the $\mathrm{HgI}_{2}$ crystal as the gamma ray scatters back out. The remaining two features, the Compton edge and the mercury escape peak, are the result of interactions of incoming gamma rays with the $\mathrm{HgI}_{2}$ detection crystal that deposit less than the full gamma-ray energy. The Compton edge is caused by incoming gamma rays that partially interact with the detector crystal, but scatter a portion back in the direction from which the gamma ray came. Scatter in other directions results in a continuum located at energies below the Compton edge. The mercury escape peak is caused by a full-energy deposition near the surface of the $\mathrm{HgI}_{2}$ detection crystal in which the $\mathrm{x}$ ray from an excited mercury atom is lost from the detector volume.

An exponential decrease in penetration of ${ }^{137} \mathrm{Cs}$ into concrete is initially assumed, with the majority of the activity near the concrete surface. Corresponding fractions of the calibration spectra are then used to compare the measured multi-scatter region of the spectrum to the levels determined by summing corresponding fractions of the penetration calibration data.

The results of the concrete-penetration-depth calibration are provided in Table 3.2. Note that the assumption is made of a penetration depth in which $95 \%$ of the activity is within the depth stated in the table. For the penetration-depth calibration, a region $44-\mathrm{keV}$ wide is used around the ${ }^{137} \mathrm{Cs}$ peak (at $662 \mathrm{keV}$ ), and a region 116-keV wide is used in the multiple-scatter (valley) region between the Compton edge (at $477 \mathrm{keV}$ ) and the mercury-escape peak (at $597 \mathrm{keV}$ ). Also note that larger values of the valleyto-peak ratio correspond either to shallow penetration depths or to higher levels of activity localized near to the surface of the concrete that mask the penetration-depth information masked by a large surface interference.

Table 3.2 contains no calculated values for penetration depth because MCNP calculations were not performed for the $\mathrm{HgI}_{2}$ detector. However, all the calibration measurements necessary to perform the MCNP calculations were taken. The plan called for performing MCNP calculations during or after the $\mathrm{HgI}_{2}$ deployment. However, the surface contamination levels found in the basin masked the information necessary to estimate the depth of contamination penetration into the concrete. As a result, there appeared to be little benefit in performing the MCNP calculations. The experimental data, however, seemed reasonable and behaved in a consistent manner with the calibration data. 


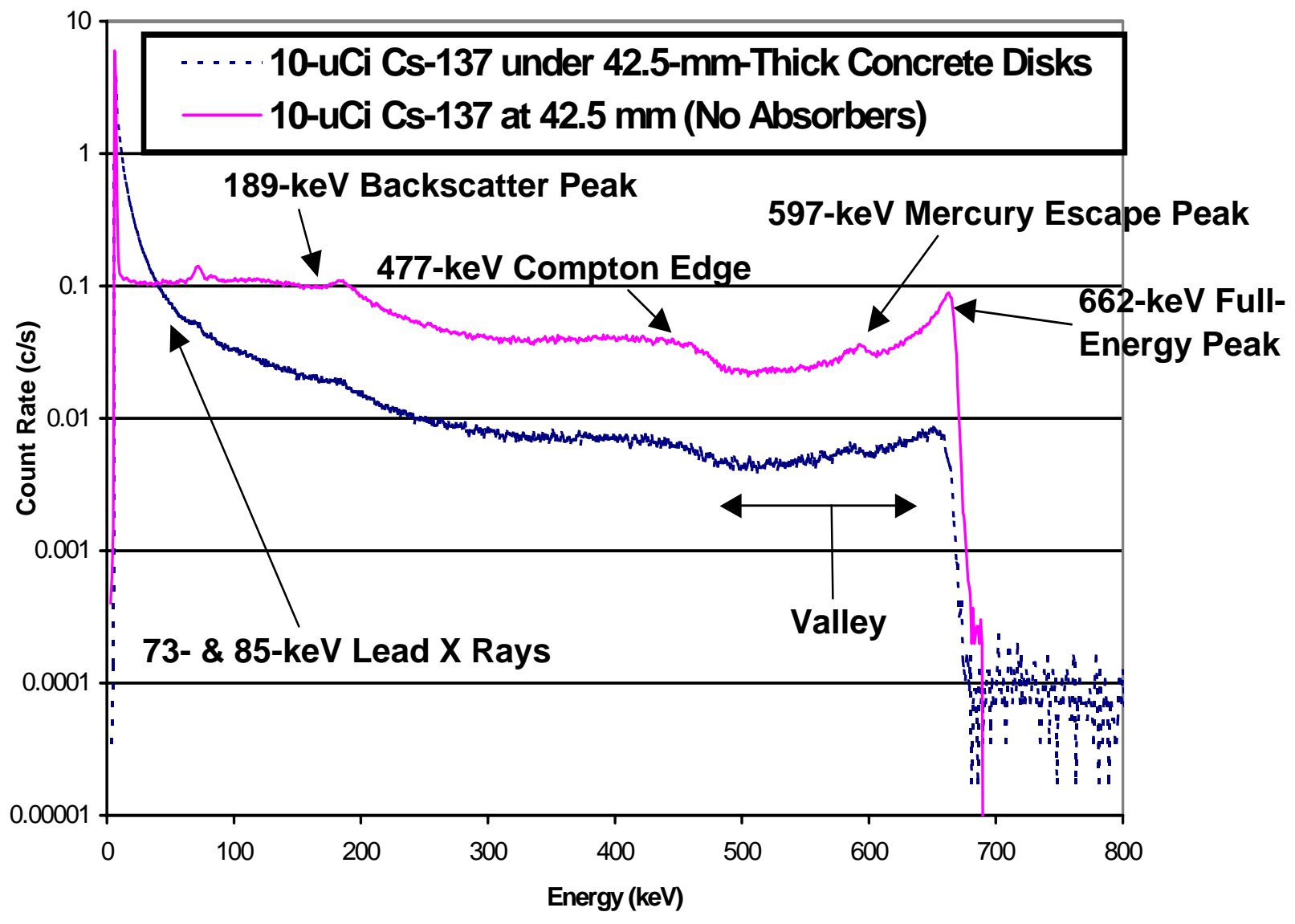

Figure 3.15. Effects of 42.5-mm Thickness of Concrete on Gamma Rays Deposited in the $\mathrm{HgI}_{2}$ Detector in the Submersible Vessel in Air

\subsubsection{Efficiency Curves}

Calibration for counting efficiency consisted of using National Institute of Science and Technology (NIST)-traceable ${ }^{137} \mathrm{Cs}$ sources to determine the gamma-ray collection efficiency, expressed as counts per gamma ray. The measured counting efficiency for the detector system is $2.2 \cdot 10^{-8} \mathrm{c} / \gamma$, which corresponds to a conversion factor of $7.0 \cdot 10^{-4} \mathrm{c} / \mathrm{s}-\mu \mathrm{Ci}$. Once the detector had been placed in the basin, the presence of the $1172-\mathrm{keV}$ gamma ray from ${ }^{60} \mathrm{Co}$ was observed in the data. The counting efficiency for ${ }^{60} \mathrm{Co}$ had not been explicitly measured with a NIST-traceable standard, so known attenuation coefficients and atomic ratios were used to estimate the counting efficiency of the $\mathrm{HgI}_{2}$ detector in the $1200-\mathrm{keV}$ range. The data predicted a counting efficiency of $56 \%$ at $1170-\mathrm{keV}$ relative to an efficiency of $100 \%$ at $660-\mathrm{keV}$, i.e., $1.2 \cdot 10^{-8} \mathrm{c} / \gamma$ or $3.9 \cdot 10^{-4} \mathrm{c} / \mathrm{s}-\mu \mathrm{Ci}$. 
Table 3.2. Mercuric-Iodide Detector in Submersible Vessel in Air in Front of Detector (10.0 $\mu \mathrm{Ci}{ }^{137} \mathrm{Cs}$ Point Source)

\begin{tabular}{|c|c|c|c||}
\hline \multirow{2}{*}{$\begin{array}{c}\text { Concrete } \\
\text { Penetration } \\
\text { Depth (mm) }\end{array}$} & \multicolumn{2}{|c|}{$\begin{array}{c}\text { Count Rate } \\
\text { (counts/second) }\end{array}$} & $\begin{array}{c}\text { Valley-to- } \\
\text { Peak Ratio }\end{array}$ \\
\cline { 2 - 4 } & $\begin{array}{c}\text { Portion of } \\
\text { Spectrum }\end{array}$ & Measured & Measured \\
\hline \multirow{2}{*}{2.5} & Valley & 12.34 & 1.996 \\
\cline { 2 - 4 } & Peak & 6.18 & \\
\hline \multirow{2}{*}{5.0} & Valley & 62.75 & 1.736 \\
\cline { 2 - 4 } & Peak & 36.15 & \\
\hline \multirow{2}{*}{7.5} & Valley & 121.0 & 1.616 \\
\cline { 2 - 4 } & Peak & 74.88 & \\
\hline \multirow{2}{*}{10.} & Valley & 179.3 & 1.566 \\
\cline { 2 - 4 } & Peak & 114.5 & \\
\hline \multirow{2}{*}{12.5} & Valley & 235.1 & 1.545 \\
\cline { 2 - 4 } & Peak & 152.1 & \\
\hline \multirow{2}{*}{15.} & Valley & 286.8 & 1.536 \\
\cline { 2 - 4 } & Peak & 186.8 & \\
\hline \multirow{2}{*}{17.5} & Valley & 334.1 & 1.532 \\
\cline { 2 - 4 } & Peak & 218.1 & \\
\hline
\end{tabular}

\subsubsection{Validation Methods and Traceability of Sources}

Calibration was performed with several different amplifier-gain settings, polarity modes, and shaping times before placement in the basin. The calibration sources were commercial "button" point sources obtained from Isotope Products Laboratories. The sources were purchased as NIST-traceable standards.

Because the polarity and gain settings were changed shortly after placement of the system to match the much higher activities actually found in the basin, another NIST-traceable standard was prepared to allow a calibration check with the system under water. The standard was prepared using a stock solution and laminated to seal the active portion in a water-tight manner. The in-basin calibration confirmed the accuracy of values provided in Table 3.3.

The calibration initially used to estimate the ${ }^{137} \mathrm{Cs}$ concentrations (based solely on total count rate) was a $10-\mu \mathrm{Ci}{ }^{137} \mathrm{Cs}(9.349-\mu \mathrm{Ci} \pm 3.0 \%$ effective) NIST traceable source. The source had been counted overnight, and, as a result, showed a large amount of activity. The source was located $2.54 \mathrm{~cm}$ from the surface of the detector, so that no activity was missed due to geometrical shielding. The appropriate geometrical correction was made to obtain the expected count rate at the surface of the detector. However, it was later determined that the pulse processing mode and gain were different for this calibration than what was used as data was collected in the basin. So, although the precision was good, the net result was a higher estimate of radioactivity associated with the basin concrete.

The calibration performed as part as the detector readiness check proved more appropriate as the pulse processing mode and gain were consistent with the settings used to collect data in the basin. This calibration was performed with a $1-\mu \mathrm{Ci}{ }^{137} \mathrm{Cs}(0.9520-\mu \mathrm{Ci} \pm 3.1 \%$ effective $)$ NIST traceable source, which was attached to the bottom of the detector and counted overnight. The count rate was maximized so that precision errors were considered less than $5 \%$. The background counts above the ${ }^{137} \mathrm{Cs}$ full-energy 
peak were negligible, so no statistical errors due to background counts were necessary. The resulting calibration value was 0.005568 counts $/$ second $(\mathrm{c} / \mathrm{s})$ or $0.01847 \mathrm{c} / \mathrm{s} / \mathrm{cm}^{2}$, using an area of $0.3167 \mathrm{~cm}^{2}$ for the $1 / 4$ inch hole in the lead collimator through which the gamma rays entered the detector.

The overall uncertainty of $11 \%$ is computed by summing the quadrature using the $3.1 \%$ source uncertainty, the 5\% positioning uncertainty, and a $10 \%$ uncertainty in reproducibility (systematic errors) for duplicate runs. Because of the large number of counts and long run times, statistical errors are limited to $2 \%$, which includes uncertainty in assuming the correct background activity level.

The overall uncertainty of $11 \%$ applies to each data value presented in Table 3.3. However, the data values in Table 3.3 have not been adjusted for the overall uncertainty, since the statistical variation is not significant between data values.

\subsubsection{Experimental Data Used to Determine the Distance between Floor Measurement Locations and Spent Fuel}

Section 2.0 of this report described the steps identified to minimize the influence of background radiation on the detection of gammas emitted by the wall and floor. Background radiation from the spent fuel on the basin floor was specifically called out as an influence to minimize. Once the $\mathrm{HgI}_{2}$ detector system was deployed, a series of measurements was performed (on February 10, 2003) to quantify the influence of the spent fuel on floor measurements. Figure 3.16 is a plot of the data collected. The starting point for the measurements was the Cubicle 4810, which was just to the left (facing north) of the spent-fuel canister in Cubicle 4710. After a 5-min count in Cubicle 4810, the detector was moved one cubicle south to Cubicle 4809, followed by 5-min counts in Cubicles 4808, 4807, 4806, 4805, and 4804. The highest activity level measured was in Cubicle 4810 adjacent to the fuel canister. The activity level dropped to a consistent level once the detector was moved at least three cubicles away from the spent-fuel canister. The data suggest that there appeared to be little benefit in maintaining a distance greater than three cubicles between the spent fuel and a specific measurement location. Given that a cubicle is approximately 10 inches from north to south and 19 inches west to east, a grid of 35 cubicles (with the measurement location in the center) was needed to eliminate additional counts in the data spectrum arising from radiation emitted by nearby spent fuel. The 35-cubicle grid is of particular importance because it relates to the difficulty of taking any measurements from a Center Bay location. All cubicles of interest in the Center Bay (note: cubicles of interest were determined by SNFP Transition Project staff) proved to be problematic in terms of the number of fuel moves required to maintain the 35-cubicle grid. The location of interest requiring the minimum number of fuel moves was cubicle 4573, which required 18 fuel moves. However, Basin resources were not available to perform the required fuel moves.

\subsubsection{Activity Levels Determined via Gamma-Ray Spectrometry}

The results of the gamma-ray analysis for the selected cubicles are summarized in Table 3.3. The activity of ${ }^{137} \mathrm{Cs}$ dominates any given spectrum. Initial reports provided during the data-collection campaign attributed all activity to ${ }^{137} \mathrm{Cs}$, which tended to over emphasize activity levels. However, the activity levels provided in Table 3.3 are based on the net peak height (and, hence, area) of the ${ }^{137} \mathrm{Cs}$ peak at $661.66 \mathrm{keV}$. Also provided are the observed activity levels for ${ }^{60} \mathrm{Co}$. As mentioned previously, no actual calibration was performed using a ${ }^{60} \mathrm{Co}$ standard, so the results are based on mercury and iodine absorption values for ${ }^{60} \mathrm{Co}$ gamma-rays relative to the ${ }^{137} \mathrm{Cs}$ gamma-ray. 


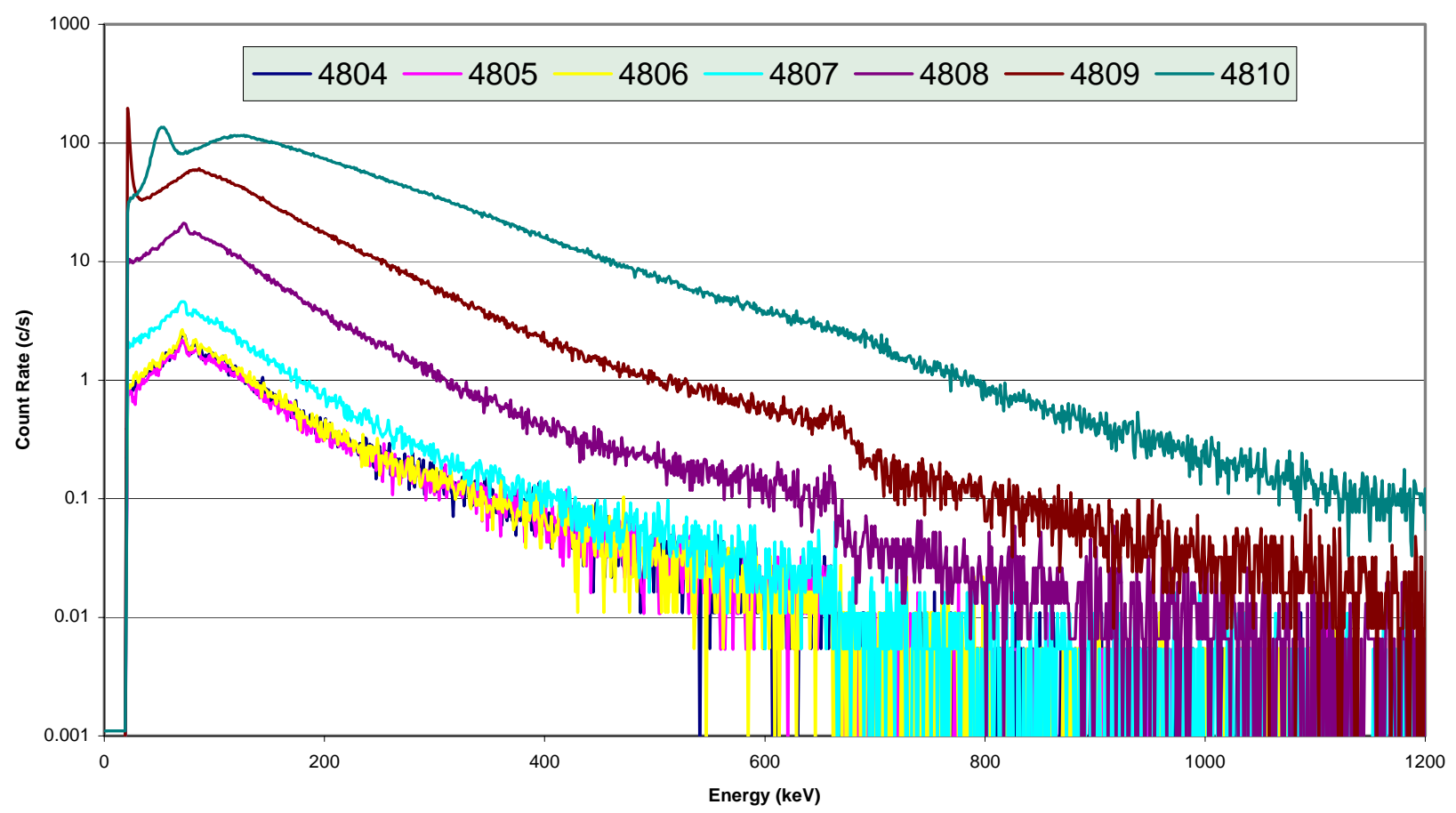

Figure 3.16. Graphical Display of the Effects on Data Quality as the Floor Detector is Moved Close to Canisters Containing Spent Fuel

Although the $723.3 \mathrm{keV}$ gamma-ray from ${ }^{154}$ Eu were not observed in any of the spectral data, upper-limit values for ${ }^{154} \mathrm{Eu}$ activity were obtained by shifting a ${ }^{137} \mathrm{Cs}$ spectrum to the $723 \mathrm{keV}$ position and adding fractions of the shifted data until a small feature was observed among the existing data. The values provided in the ${ }^{154} \mathrm{Eu}$ data column in Table 3.3, therefore, represent more than the greatest amount of ${ }^{154} \mathrm{Eu}$ that could be present without being detected.

Another data column represents non-specific activity present in the data spectra. This represents additional counts in a given spectrum that exceed the count rate explained by the presence of ${ }^{137} \mathrm{Cs}$ and ${ }^{60} \mathrm{Co}$. The nonspecific activity is assumed to be explained by the presence of radioactive species, such as ${ }^{90} \mathrm{Sr}$, that emit beta particles and conversion electrons that give rise to bremsstrahlung radiations. Although the data confirms the presences of bremsstrahlung due to ${ }^{90} \mathrm{Sr}$, the level of ${ }^{90} \mathrm{Sr}$ cannot be quantified because the bremmsstrahlung intensity is totally related to matrix effects (i.e., contact with uranium).

As mentioned previously, the underwater surfaces of the basin were assumed to be primarily contaminated with ${ }^{137} \mathrm{Cs}$ at levels near $12.9 \mu \mathrm{Ci} / \mathrm{cm}^{2}$, as found on the 1980 core samples (Bechtold 1981). The floor detector system measured ${ }^{137} \mathrm{Cs}$ activity levels significantly higher than expected (see Table 3.3 and Figure 3.17). Activity levels of ${ }^{137} \mathrm{Cs}$ on the basin floor ranged from a low of $85 \mu \mathrm{Ci} / \mathrm{cm}^{2}$ to a high $3800 \mu \mathrm{Ci} / \mathrm{cm}^{2}$, depending on location. The variation in activity levels were measured from one bay of the basin to another, from one cubicle to another in the same bay, and from one side of a cubicle to the other side of the same cubicle. The highest and lowest activity levels measured were found in the same cubicle (Cubicle 2211 West and East). 
Table 3.3. Summary of NDE Measurements on KE Basin Floor and Wall

\begin{tabular}{|c|c|c|c|c|c|c|c|c|c|c|c|c|c|c|c|}
\hline \multirow{2}{*}{ Run } & \multicolumn{2}{|c|}{ Cubicle Position } & \multicolumn{5}{|c|}{ Measured Activity $\left(\mu \mathrm{Ci} / \mathrm{cm}^{2}\right)$} & \multirow{2}{*}{$\begin{array}{c}\text { Water } \\
\text { Pressure } \\
\text { (psi) }\end{array}$} & \multirow{2}{*}{ Brushed } & \multicolumn{2}{|c|}{1994 Canister Present } & \multirow{2}{*}{$\begin{array}{c}\text { Estimated } \\
\text { Sludge Depth } \\
\quad \text { (inches) }\end{array}$} & \multicolumn{2}{|c|}{$\begin{array}{c}\text { General Basin } \\
\text { Position }\end{array}$} & \multirow{2}{*}{ Comments } \\
\hline & No. & Side & $\begin{array}{l}\text { Counts/ } \\
\text { Second }\end{array}$ & ${ }^{137} \mathrm{Cs}$ & ${ }^{154} \mathbf{E} \mathbf{u}^{(b)}$ & ${ }^{60} \mathrm{Co}$ & Other $^{(a)}$ & & & $\begin{array}{c}\text { Fuel } \\
\text { Cond./Key }\end{array}$ & $\begin{array}{c}\text { Canister } \\
\text { Bottom }\end{array}$ & & Bay & $\begin{array}{c}\text { North or } \\
\text { South }\end{array}$ & \\
\hline 1 & 5209 & East & 8054 & $>870$ & N/A & N/A & N/A & 110 & No & Empty & N/A & 1.3 & West & South & $\begin{array}{l}\text { Short duration data point, } \\
\text { adjusted height of spray wand }\end{array}$ \\
\hline 2 & 5209 & East & 4316 & 504 & $<2.0$ & 0.70 & $<20$ & 400 & No & Empty & N/A & 1.3 & West & South & \\
\hline 3 & 5209 & East & 4097 & 479 & $<2.0$ & 0.80 & $<20$ & 1300 & No & Empty & N/A & 1.3 & West & South & \\
\hline 4 & 5211 & East & 2616 & 226 & $<1.0$ & 14 & 69 & 110 & No & Fair/11897 & $?$ & 1.3 & West & South & Moved ID disks \\
\hline 5 & 5211 & East & 2359 & 218 & $<1.0$ & 2.5 & 76 & 1250 & No & Fair/11897 & $?$ & 1.3 & West & South & \\
\hline 6 & 6040 & East & 1420 & 152 & $<0.5$ & 0.65 & $<20$ & 110 & No & Fair/11897 & $?$ & 0.6 & West & Mid & \\
\hline 7 & 6040 & East & 1341 & 147 & $<0.5$ & 0.62 & $<20$ & 1100 & No & Fair/11897 & $?$ & 0.6 & West & Mid & \\
\hline 8 & 5209 & East & 4224 & 499 & $<2.0$ & 0.75 & $<20$ & 110 & No & Empty & N/A & 1.3 & West & South & Verify detector data \\
\hline 9 & 5209 & West & 3312 & 334 & $<1.5$ & 0.64 & 57 & 110 & No & Empty & N/A & 1.3 & West & South & \\
\hline 10 & 2209 & West & 1681 & 195 & $<1.0$ & 0.53 & $<20$ & 110 & No & Fair/0602 & Al-Screened & 1.9 & East & South & $\begin{array}{l}\text { Moved ID disk and battery. Next } \\
\text { to wall. }\end{array}$ \\
\hline 11 & 2209 & West & 1336 & 149 & $<0.5$ & 0.54 & $<20$ & 1275 & No & Fair/0602 & Al-Screened & 1.9 & East & South & \\
\hline 12 & 2209 & West & 1463 & 179 & $<1.0$ & 0.47 & $<20$ & 1250 & Yes & Fair/0602 & Al-Screened & 1.9 & East & South & \\
\hline 13 & 2211 & West & 34919 & $>3800$ & N/A & $\mathrm{N} / \mathrm{A}$ & N/A & 110 & No & $\begin{array}{l}\text { Very } \\
\text { Bad/0456 }\end{array}$ & Open Screened & 1.5 & East & South & Moved ID disk. Next to wall. \\
\hline 14 & 2211 & West & 33326 & $>3600$ & N/A & N/A & N/A & 1250 & No & \begin{tabular}{|l} 
Very \\
Bad/0456
\end{tabular} & Open Screened & 1.5 & East & South & \\
\hline 15 & 2211 & West & 32004 & $>3500$ & N/A & N/A & N/A & 110 & Yes & \begin{tabular}{|l} 
Very \\
Bad/0456 \\
\end{tabular} & Open Screened & 1.5 & East & South & High pressure wash not available \\
\hline 16 & 2211 & West & 32903 & $>3600$ & N/A & $\mathrm{N} / \mathrm{A}$ & N/A & 150 & Yes & \begin{tabular}{|l|} 
Very \\
Bad/0456
\end{tabular} & Open Screened & 1.5 & East & South & Re-brush and wash \\
\hline 17 & 2211 & East & 783 & 86 & $<0.5$ & 0.51 & 42 & 150 & No & \begin{tabular}{|l} 
Very \\
Bad/0456
\end{tabular} & Open Screened & 1.5 & East & South & \\
\hline 18 & 2211 & East & 766 & 85 & $<0.5$ & 0.44 & 40 & 1200 & No & \begin{tabular}{|l} 
Very \\
Bad/0456
\end{tabular} & Open Screened & 1.5 & East & South & \\
\hline 19 & 2211 & East & 763 & 89 & $<1.0$ & 0.73 & 38 & 150 & Yes & \begin{tabular}{|l|} 
Very \\
Bad/0456
\end{tabular} & Open Screened & 1.5 & East & South & Two-hour count \\
\hline 20 & 2209 & West & 1591 & 213 & $<1.0$ & 0.44 & $<20$ & 110 & No & Fair/0602 & Al-Screened & 1.9 & East & South & Brushed previously. \\
\hline 21 & 5209 & East & 3933 & 475 & $<2.0$ & 0.33 & $<20$ & 110 & No & Empty & N/A & 1.3 & West & South & \\
\hline
\end{tabular}

(a) Includes other radionuclides such as ${ }^{90} \mathrm{Sr}$ and ${ }^{40} \mathrm{~K}$.

(b) Upper limit values for ${ }^{154} \mathrm{Eu}$.

\begin{tabular}{||l|c|c|c|c|c|c|c|c||}
\hline \multirow{2}{*}{ Table Location } & \multirow{2}{*}{$\begin{array}{c}\text { Height from } \\
\text { Floor } \\
\text { (inches) }\end{array}$} & $\begin{array}{c}\text { Counts/ } \\
\text { Second }\end{array}$ & ${ }^{137} \mathbf{C s}$ & ${ }^{154} \mathbf{E u}$ & ${ }^{60} \mathbf{C o}$ & \multirow{2}{*}{ Other ${ }^{\text {(a) }}$} & \multirow{2}{*}{ Brushed } & \multirow{2}{*}{ Comment } \\
\hline $\begin{array}{l}\text { Wall adjacent to where } \\
\text { cubicles 2213 and 2214 meet }\end{array}$ & 28.5 & 3437 & 368 & $<1.5$ & 0.07 & 61 & \multirow{2}{*}{ No } & East Bay on partition wall \\
\hline Same & Same & 3438 & 360 & $<1.5$ & 0.08 & 69 & No & Same \\
\hline Same & Same & 2479 & 176 & $<1.5$ & 0.06 & 133 & Yes & Same \\
\hline
\end{tabular}




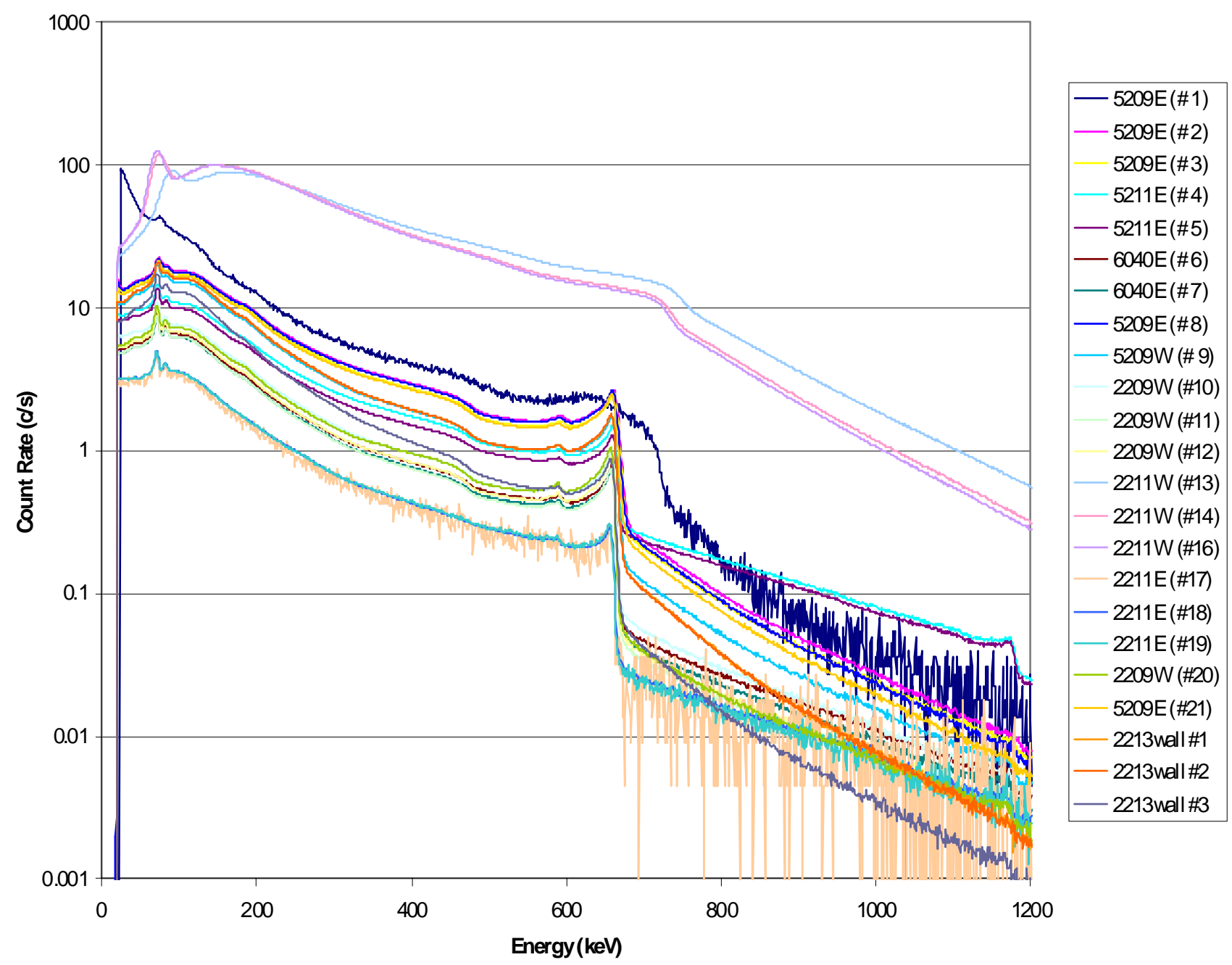

Figure 3.17. Graphical Display of All Significant Data in the Data-Collection Campaign for the K East Basin Floor

Attempts to clean the floor or wall using high-pressure wash followed with a stainless steel brushing (and a final wash) appeared to have only modest benefit. Activity levels of ${ }^{137} \mathrm{Cs}$ levels on the basin wall before cleaning were 360 to $368 \mu \mathrm{Ci} / \mathrm{cm}^{2}$ and $176 \mu \mathrm{Ci} / \mathrm{cm}^{2}$ after cleaning with a stainless steel brush. The higher than expected ${ }^{137} \mathrm{Cs}$ activity level made it virtually impossible to quantify depth of contaminant penetration into the concrete. Although the depth-of-penetration could not be quantified, the valley-to-peak ratio in each spectra collected (see Figure 3.17) suggested that the majority of the observed radionuclide activity is on or near the surface of the concrete (within 2-3 $\mathrm{mm}$ of the surface).

It is important to note that determination of contaminant penetration into the concrete is not solely due to the total activity level, but is more precisely determined by the ratio of surface activity to the activity embedded in the concrete. As long as the surface activity is significantly higher than the activity found below $2-3 \mathrm{~mm}$ in the concrete, it will be difficult to quantify depth of contaminant penetration into the concrete. For example, if 10 $\mu \mathrm{Ci} / \mathrm{cm}^{2}$ of ${ }^{137} \mathrm{Cs}$ has penetrated $3 \mathrm{~mm}$ or more into the concrete, that level of activity would be masked by surface activity levels of $40 \mu \mathrm{Ci} / \mathrm{cm}^{2}$ of ${ }^{137} \mathrm{Cs}$ or greater. 


\subsubsection{Quality Assurance}

The NDE work was performed consistent to PNNL Quality Assurance (QA) Project Plan 43883-QAPjP, Rev. 0 and the QA requirements in the FH SAP (Baker et al. 2002).

The sources used in the laboratory calibration of the $\mathrm{HgI}_{2}$ detector system were NIST-traceable commercial "button" point sources obtained from Isotope Products Laboratories. The first laboratory calibration verified and optimized detector operation, including identification of hardware, using $1-\mu \mathrm{Ci}$ and $10-\mu \mathrm{Ci}{ }^{137} \mathrm{Cs}$ point sources. The second laboratory calibration used the $10-\mu \mathrm{Ci}{ }^{137} \mathrm{Cs}$ point source to calibrate the detector responsiveness to source activity through various thicknesses of concrete (up to $17.5 \mathrm{~mm}$ ). The third laboratory calibration used the $1-\mu \mathrm{Ci}{ }^{137} \mathrm{Cs}$ point source as part of the detector underwater readiness check. This last calibration proved to be the most appropriate for use in performing data reduction and interpretation of the measurements taken in the basin.

The in-basin calibration used a $10-\mu \mathrm{Ci}{ }^{137} \mathrm{Cs}$ source (radiation standard $56595-101-\mathrm{B}$ ), which was prepared by E. A. Lepel on January 24, 2003. When the source was used on May 28, 2003, the decay-corrected activity was $9.323 \mu \mathrm{Ci} \pm 1.06 \%$.

As mentioned previously, a technical data package containing the QA Project Plan, source certificates of calibration, submerged fan jet analysis, the standard operating procedure for collecting data from floor locations, location measurement log sheets, raw data files, procedure for ${ }^{137} \mathrm{Cs}$ activity calculation, Excel spreadsheets, and spectra for each location measured has been provided to FH (R.B. Baker). 


\subsection{References}

Baker RB. 1995. Summary Status of K Basins Sludge Characterization. WHC-SD-SNF-TI-006, Westinghouse Hanford Company, Richland, WA.

Baker RB. 2001. Estimated Volumes of Sludge in the Hanford K East and K West Basins. HNF-8118, Rev. 0, Fluor Hanford, Richland, WA.

Baker RB, BJ Makenas, and SN Schlahta. 2002. Sampling and Analysis Plan for Characterization of Contamination in the Walls and Floor of the Hanford K Basins. SNF-10845, Fluor Hanford, Richland, WA.

Bechtold DB. 1981. KE Fuel Storage Facility Activity Mapping in Support of Exposure Reduction. UNI-1697, United Nuclear Industries, Richland, WA.

Briesmeister JF, Ed. 2000. MCNP- A General Monte Carlo N-Particle Transport Code, Version 4C. LA-13709-M (April, 2000), Los Alamos, NM.

Huang FF, and FW Moore. 1997. Dose Reduction Improvements in Storage Basins of Spent Nuclear Fuel. HNF-SA-3158-FP, Fluor Daniel Northwest, Richland, WA.

Johnson AB, and S Burke. 1995. K Basin Corrosion Program Report. WHC-EP-0877, Westinghouse Hanford Company, Richland, WA.

Makenas BJ, and RB Baker. 2002. Data Quality Objectives for K Basin Wall Characterization.

SNF-9853, Fluor Hanford, Richland, WA.

Schmidt AJ, and MR Elmore. 2002. Settling Test Using Simulants to Evaluate Uranium Metal Distribution in K Basin Sludge. PNNL-13854, Pacific Northwest National Laboratory, Richland, WA.

Vargo GJ, JS Durham, EE Hickey, PS Stansbury, and GR Cicotte. 1994. Review of ALARA Plan for Activities at the 105 K-East Fuel Storage Basin. PNL-9826, Rev. 2, Pacific Northwest Laboratory, Richland WA. 


\section{Appendix A}

Manufacturer Specification for HPGe Detector 


\section{Standard Electrode Coaxial Ge Detectors}

\section{Features}

- Wide range of efficiencies

- High resolution - good peak shape

- Excellent timing resolution

- High energy rate capability

- Diode FET protection

- Warm-up/HV shutdown

- High rate indicator

\section{Description}

The conventional coaxial germanium detector is often referred to as Pure Ge, HPGe, Intrinsic Ge, or Hyperpure Ge. Regardless of the superlative used, the detector is basically a cylinder of germanium with an n-type contact on the outer surface, and a p-type contact on the surface of an axial well. The germanium has a net impurity level of around $10^{10}$ atoms/cc so that with moderate reverse bias, the entire volume between the electrodes is depleted, and an electric field extends across this active region. Photon interaction within this region produces charge carriers which are swept by the electric field to their collecting electrodes, where a charge sensitive preamplifier converts this charge into a voltage pulse proportional to the energy deposited in the detector.

The $\mathrm{n}$ and $\mathrm{p}$ contacts, or electrodes, are typically diffused lithium and implanted boron respectively. The outer n-type diffused lithium contact is about $0.5 \mathrm{~mm}$ thick. The inner contact is about $0.3 \mu \mathrm{m}$ thick. A surface barrier may be substituted for the implanted boron with equal results.

The Canberra Coaxial Ge detector can be shipped and stored without cooling. However, long term stability is best preserved by keeping the detector cold. Like all germanium detectors, it must be cooled when it is used to avoid excessive thermally-generated

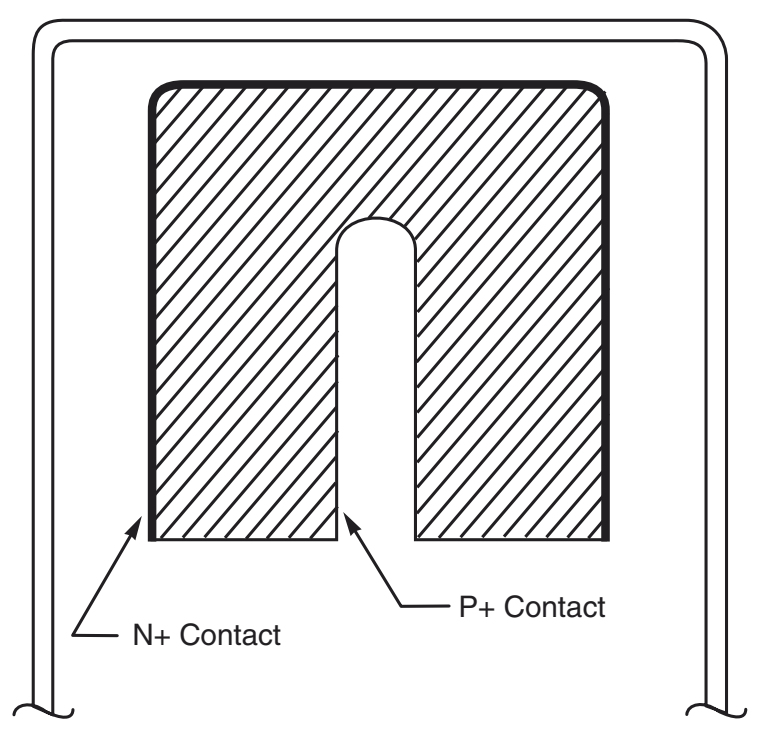

Coaxial Ge Detector Configuration

leakage current. The non-perishable nature of this detector widens the application of $\mathrm{Ge}$ spectrometers to include field use of portable spectrometers.

The useful energy range of the Coaxial Ge detector is $50 \mathrm{keV}$ to more than $10 \mathrm{MeV}$. The resolution and peak shapes are excellent and are available over a wide range of efficiencies. A list of available models is given in the accompanying table.

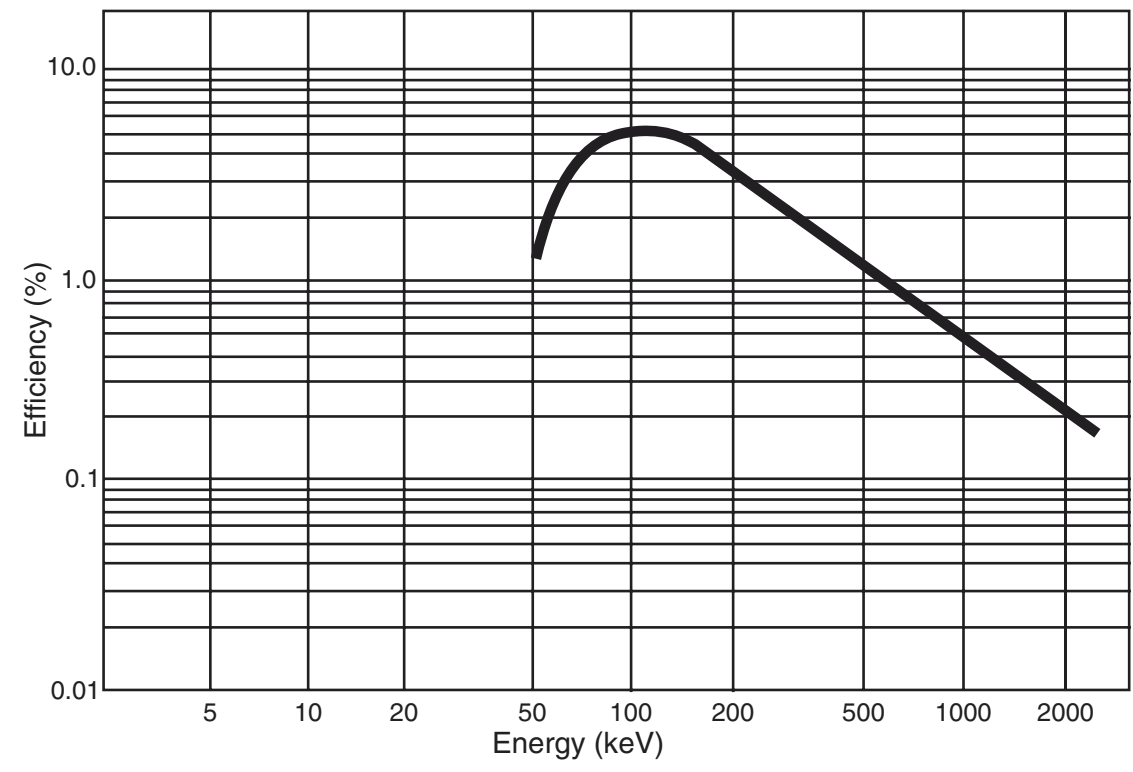

Typical Absolute Efficiency Curve for 15\% Detector (25 cm detector to source spacing) 
COAXIAL GERMANIUM DETECTOR

General Specifications and Information

Standard configuration includes:

- Vertical slimline cryostat with 30 liter Dewar

- Model 2002C Preamplifier with 3 meter bias, high voltage

inhibit, signal, and power cables

Specify cryostat option from options price list

Resolution at $122 \mathrm{keV}$ is a typical value, not a specification limit.

\begin{tabular}{|c|c|c|c|c|c|}
\hline \multirow[b]{2}{*}{$\begin{array}{l}\text { Model } \\
\text { Number }\end{array}$} & \multirow[b]{2}{*}{$\begin{array}{l}\text { Relative } \\
\text { Efficiency }\end{array}$} & \multicolumn{3}{|c|}{ Resolution } & \multirow[b]{2}{*}{$\begin{array}{c}\text { Peak/ } \\
\text { Compton }\end{array}$} \\
\hline & & $\begin{array}{l}\text { FWHM } \\
(122 \mathrm{keV})\end{array}$ & $\begin{array}{c}\text { FWHM } \\
(1332 \mathrm{keV})\end{array}$ & $\begin{array}{c}\text { FWTM } \\
(1332 \mathrm{keV})\end{array}$ & \\
\hline GC1018 & 10 & 0.8 & 1.8 & 3.4 & 38 \\
\hline GC1019 & 10 & 0.9 & 1.9 & 3.7 & 36 \\
\hline GC1020 & 10 & 0.9 & 2.0 & 4.0 & 34 \\
\hline GC1518 & 15 & 0.8 & 1.8 & 3.4 & 44 \\
\hline GC1519 & 15 & 0.9 & 1.9 & 3.7 & 42 \\
\hline GC1520 & 15 & 0.9 & 2.0 & 4.0 & 40 \\
\hline GC2018 & 20 & 0.8 & 1.8 & 3.4 & 50 \\
\hline GC2019 & 20 & 0.9 & 1.9 & 3.7 & 48 \\
\hline GC2020 & 20 & 0.9 & 2.0 & 4.0 & 46 \\
\hline GC2518 & 25 & 0.8 & 1.8 & 3.4 & 54 \\
\hline GC2519 & 25 & 0.9 & 1.9 & 3.7 & 52 \\
\hline GC2520 & 25 & 0.9 & 2.0 & 4.0 & 50 \\
\hline GC3018 & 30 & 0.8 & 1.8 & 3.4 & 58 \\
\hline GC3019 & 30 & 0.9 & 1.9 & 3.7 & 56 \\
\hline GC3020 & 30 & 1.0 & 2.0 & 4.0 & 54 \\
\hline GC3518 & 35 & 0.9 & 1.8 & 3.4 & 60 \\
\hline GC3519 & 35 & 0.9 & 1.9 & 3.7 & 58 \\
\hline GC3520 & 35 & 1.0 & 2.0 & 4.0 & 56 \\
\hline GC4018 & 40 & 0.9 & 1.8 & 3.4 & 62 \\
\hline GC4019 & 40 & 1.0 & 1.9 & 3.7 & 58 \\
\hline GC4020 & 40 & 1.1 & 2.0 & 4.0 & 54 \\
\hline GC4519 & 45 & 1.0 & 1.9 & 3.7 & 62 \\
\hline GC4520 & 45 & 1.1 & 2.0 & 4.0 & 58 \\
\hline GC4521 & 45 & 1.2 & 2.1 & 4.2 & 54 \\
\hline GC5019 & 50 & 1.0 & 1.9 & 3.7 & 64 \\
\hline GC5020 & 50 & 1.1 & 2.0 & 4.0 & 60 \\
\hline GC5021 & 50 & 1.2 & 2.1 & 4.2 & 56 \\
\hline
\end{tabular}

\begin{tabular}{||c|c|c|c|c|c||}
\hline & & \multicolumn{3}{|c|}{ Resolution } & \\
\cline { 3 - 5 } $\begin{array}{c}\text { Model } \\
\text { Number }\end{array}$ & $\begin{array}{c}\text { Relative } \\
\text { Efficiency }\end{array}$ & $\begin{array}{c}\text { FWHM } \\
\text { (122 keV) }\end{array}$ & $\begin{array}{c}\text { FWHM } \\
\text { (1332 keV) }\end{array}$ & $\begin{array}{c}\text { FWTM } \\
\text { (1332 keV) }\end{array}$ & $\begin{array}{c}\text { Peak/ } \\
\text { Compton }\end{array}$ \\
\hline GC5520 & 55 & 1.1 & 2.0 & 3.8 & 64 \\
GC5521 & 55 & 1.2 & 2.1 & 4.1 & 60 \\
GC5522 & 55 & 1.3 & 2.2 & 4.4 & 56 \\
GC6020 & 60 & 1.1 & 2.0 & 3.8 & 66 \\
GC6022 & 60 & 1.2 & 2.2 & 4.4 & 60 \\
GC6520 & 65 & 1.1 & 2.0 & 3.8 & 68 \\
GC6522 & 65 & 1.2 & 2.2 & 4.4 & 62 \\
GC7020 & 70 & 1.1 & 2.0 & 3.8 & 70 \\
GC7022 & 70 & 1.2 & 2.2 & 4.4 & 64 \\
GC7520 & 75 & 1.1 & 2.0 & 3.8 & 72 \\
GC7522 & 75 & 1.2 & 2.2 & 4.4 & 66 \\
GC8021 & 80 & 1.1 & 2.1 & 4.0 & 72 \\
GC8023 & 80 & 1.2 & 2.3 & 4.6 & 68 \\
GC8521 & 85 & 1.1 & 2.1 & 4.0 & 74 \\
GC8523 & 85 & 1.2 & 2.3 & 4.6 & 68 \\
GC9021 & 90 & 1.2 & 2.1 & 4.0 & 76 \\
GC9023 & 90 & 1.3 & 2.3 & 4.6 & 70 \\
GC9521 & 95 & 1.2 & 2.1 & 4.0 & 78 \\
GC9523 & 95 & 1.3 & 2.3 & 4.6 & 72 \\
GC10021 & 100 & 1.2 & 2.1 & 4.0 & 80 \\
GC10023 & 100 & 1.3 & 2.3 & 4.6 & 74 \\
\hline & & & & & \\
\hline
\end{tabular}

Consult the factory for availability of larger detectors. 


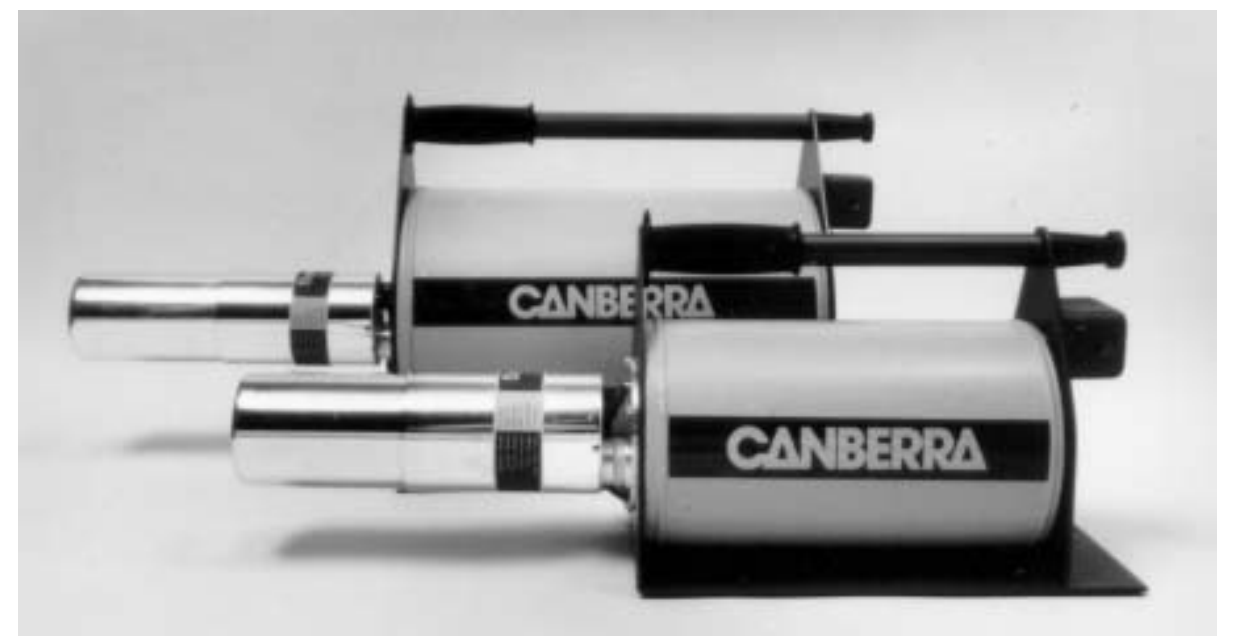

\section{Features}

- Operation in any orientation

- Light weight aluminum construction

- Slimline detector/preamplifier configuration

- Long holding time

- Warm-up sensor-bias disable

\section{Description}

For applications requiring both portability and flexibility of use, the MAC (multi-attitude cryostat) is the answer. The unique fill and vent system employed by the MAC allows operation of the detector in any orientation without $\mathrm{LN}_{2}$ spillage even when the Dewar is full. The small size, light weight, and ruggedness of the unit permit use of the unit in field conditions. The slimline detector chamber allows the unit to be shielded very effectively for use in low level counting applications.

The MAC detector consists of a Dewar having two fill and vent ports arranged so that one of the ports is the vent, regardless of the Dewar's orientation. This allows the Dewar to be operated in the horizontal position, vertically uplooking, or vertically downlooking, without loss of $\mathrm{LN}_{2}$.

A single port version of the MAC and Big MAC is available on special order. This version has half the capacity and holding time of the standard product. A gravity-feed supply Dewar/stand is available for the single port cryostat. The single port cryostat is compatible with other brands, and it holds $\mathrm{LN}_{2}$ in all orientations which may be important in some applications, e.g. for use in a submarine (see Canberra Model 7411).

The detector/preamp includes a sensor which provides a signal when the $\mathrm{LN}_{2}$ is depleted. This output can be used to shut down the bias supply, to operate an alarm, or both.

The standard MAC features Canberra's slimline cryostat option in which a Canberra preamplifier is packaged behind the detector chamber within the confines of the $80 \mathrm{~mm}$ diameter snout. The slimline cryostat allows the detector to be installed in a shield with very little difficulty and with efficient use of shielding material. The snout is long enough to reach through $10-15 \mathrm{~cm}$ of shielding material and still accommodate Marinelli beaker samples.

A flanged version of the MAC is also available. This version makes use of a conventional box style preamplifier having bulkhead connectors (rather than pigtail connectors) and is somewhat more compact than the slimline version.

The MAC comes with detachable carrying handle assembly. With the carrying handle assembly removed, there are no obstructions beyond the outer diameter of the Dewar, and the unit can be readily installed in other scientific apparatus such as whole-body counters, scattering chambers or low-level counting systems.

Both manual and automatic refill systems are available for use with the MAC. Since the MAC has separate fill and vent ports, the $\mathrm{LN}_{2}$ supply and the vent lines can be made gas tight, thus avoiding the hazards of cold $\mathrm{N}_{2}$ or $\mathrm{LN}_{2}$ to either personnel or adjacent equipment.

The MAC is available as an option with most of the High Purity Germanium detectors offered by Canberra. Consult the Canberra Catalog for information on the wide variety of detectors that are available from Canberra.

\section{Specifications \\ MAC}

WEIGHT - $5.1 \mathrm{~kg}(11.2 \mathrm{lb})$ empty; $7.1 \mathrm{~kg}(15.6 \mathrm{lb})$ full

$\mathrm{LN}_{2}$ CAPACITY - 2.5 liters

HOLDING TIME - 2 days (typical detector size)

COOL DOWN TIME - 2 hours, typically

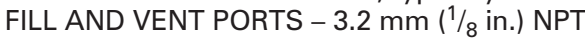

BIG MAC

WEIGHT - $7.9 \mathrm{~kg}(17.5 \mathrm{lb})$ empty; $13.6 \mathrm{~kg}(30 \mathrm{lb})$ full

$\mathrm{LN}_{2}$ CAPACITY - 7.0 liters

HOLDING TIME - 5 days (typical detector size)

COOL DOWN TIME - 2 hours, typically

FILL AND VENT PORTS $-3.2 \mathrm{~mm}(1 / 8$ in.) NPT

OPTIONS

Model 7415 Detector Lift Mechanism (for Canberra shields) 


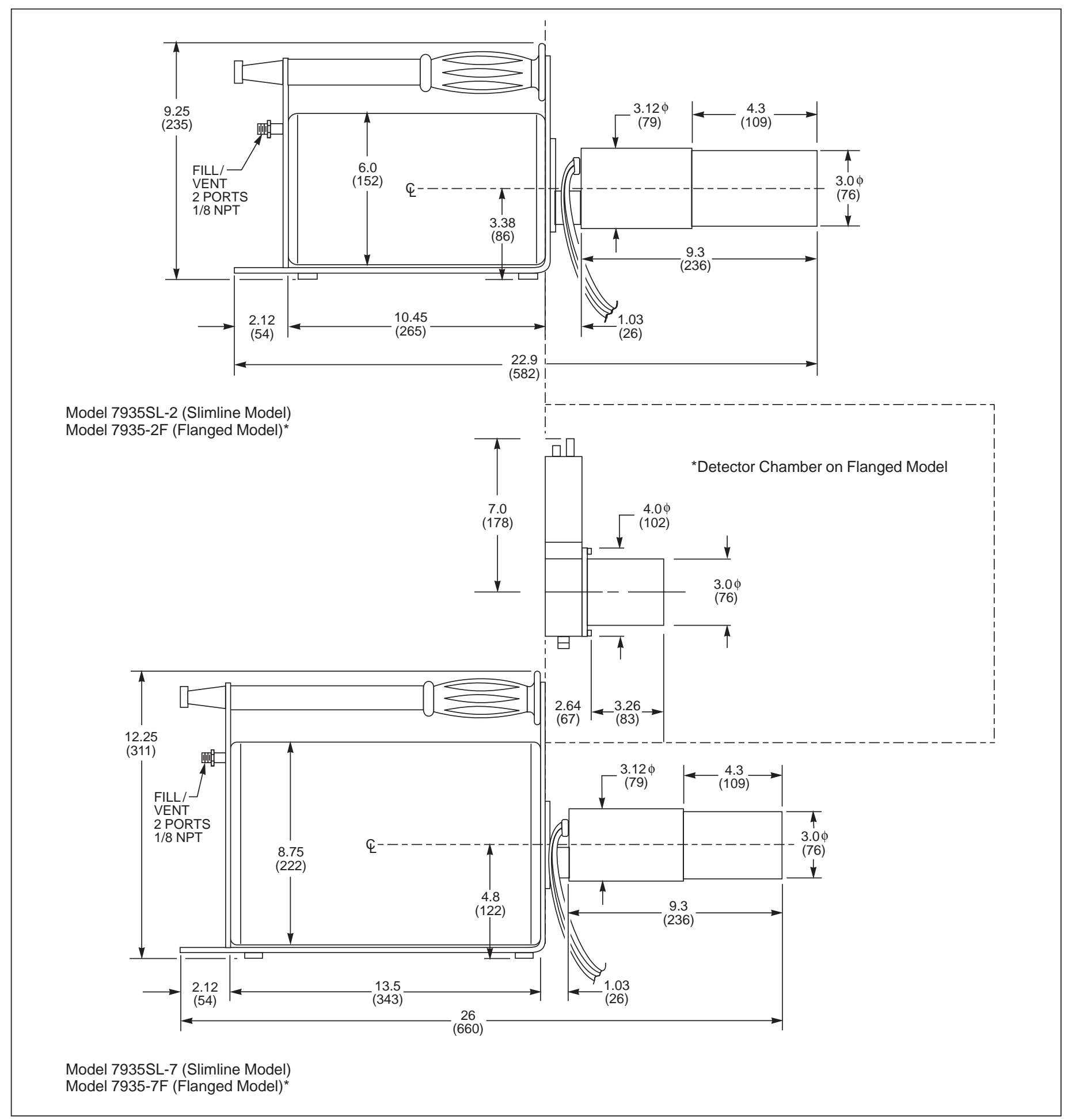

End cap dimensions depend on detector size. The chart shows the typical efficiency range vs. end-cap diameter. End cap lengths are also greater for larger detectors. Consult the factory if end-cap size is critical in your application.

\begin{tabular}{||c|cc||}
\hline Rel. Efficiency (\%) & \multicolumn{2}{|c|}{ Diameter in. (mm) } \\
\hline$\leq 40$ & 3.0 & $(76)$ \\
$40-50$ & 3.25 & $(83)$ \\
$50-70$ & 3.50 & $(89)$ \\
$70-100$ & 3.75 & $(95)$ \\
$\geq 100$ & 4.0 & $(102)$ \\
\hline
\end{tabular}




\section{Appendix B}

\section{Sketch of HPGe Detector System Submersible Vessel}




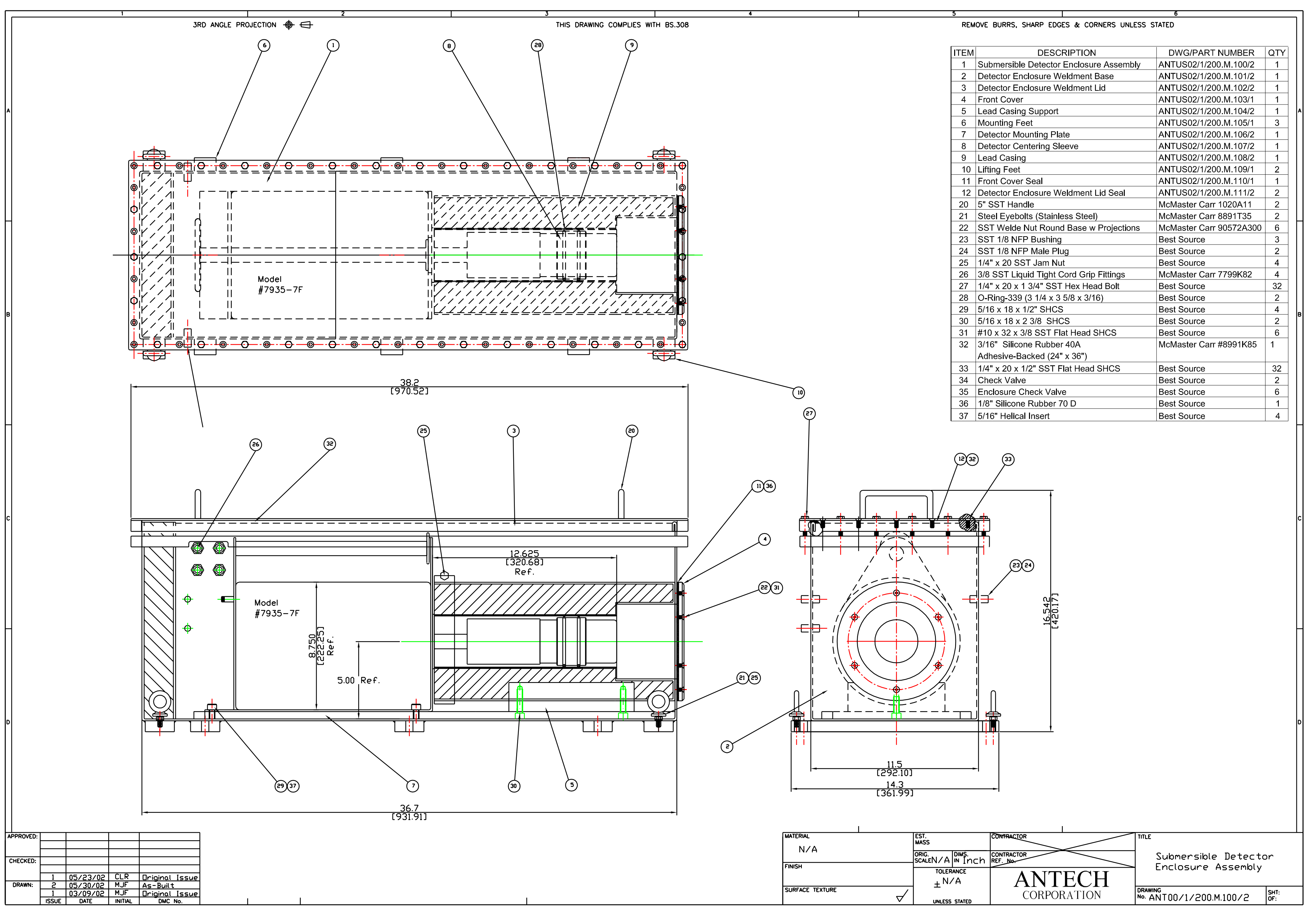




\section{Appendix C}

\section{Manufacturer Specification for $\mathrm{HgI}_{2}$ Detector}




\section{Mercuric}

\section{lodide}

\section{Detectors}

\section{๑}

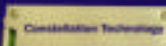

Memeumr $x$

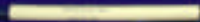

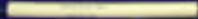
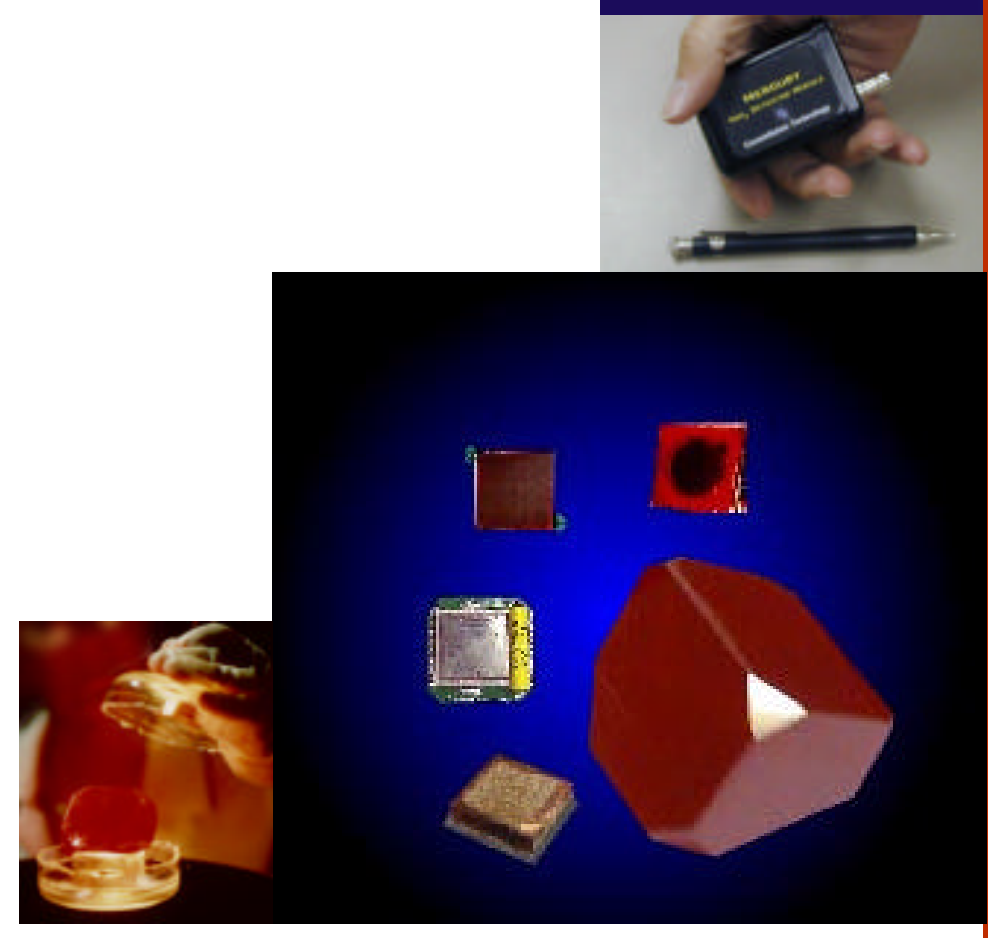

Constellation Technology Corporation

7887 Bryan Dairy Rd., Suite 100

$$
\text { Largo, FL } 33777
$$

Phone: 727-547-0600 * Fax: 727-545-6150 * Toll Free 800-335-7355 info@contech.com 


\section{Constellation's Mercuric lodide Detectors}

Recent developments at Constellation have led to dramatic improvements in the resolution performance, efficiency, stability, longevity, and durability of mercuric iodide $\left(\mathrm{HgI}_{2}\right)$ $\mathrm{x}$-ray and gamma ray spectrometers. $\mathrm{HgI}_{2}$ detectors are perfect for use in portable applications and laboratory situations where the expense of high purity germanium (HPGe) systems or the low resolution afforded by scintillators such as sodium iodide are unacceptable.

In applications that require spectral resolution better than can be achieved with sodium iodide detectors, Constellation's $\mathrm{HgI}_{2}$ detectors might provide an excellent alternative to very expensive, cumbersome, but ultra-high-resolution HPGe systems.

\section{High Resolution}

Because of its low leakage current and high band gap, $\mathrm{HgI}_{2}$ detectors have excellent spectral resolution. $\mathrm{HgI}_{2}$ detectors can replace liquid nitrogen-cooled germanium detectors in many applications and have better performance over a broader energy range than cadmium zinc telluride (CZT).

$\mathrm{HgI}_{2}$ can replace dual CZT/silicon in wide energy range $\mathrm{x}$-ray fluorescence systems because $\mathrm{HgI}_{2}$ has equal or better performance over the required energy range.

Constellation has x-ray detectors in stock that achieve resolution less than 300 eV FWHM @ 5.9 keV.

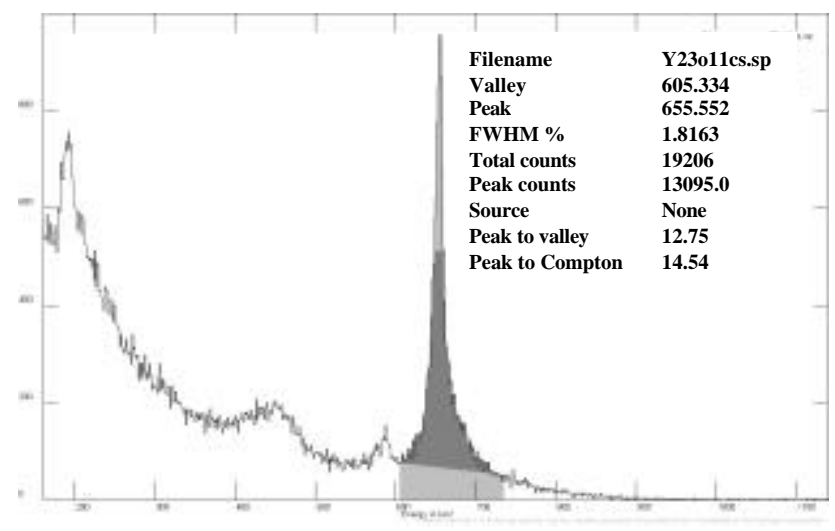

Energy spectra for ${ }^{137} \mathrm{Cs}(662 \mathrm{keV})$ emission

\section{FWHM vs. "UHWHM"}

Full Width at Half Maximum (FWHM) is a standard way of reporting peak resolution. The FWHM is a measure of the width of a peak at half its maximum value. FWHM is considered a reasonable measure but some manufacturers of HPGe detectors for example might even report the Full Width at Tenth Max (FWTM) value, which is a measure of the peak width at one tenth of the maximum peak value.

HPGe detectors and NaI detectors provide Gaussian peakshapes - the peaks are roughly symmetric around the peak center. Unlike $\mathrm{NaI}$ and $\mathrm{HPGe}$ detectors, $\mathrm{HgI}_{2}$ and $\mathrm{CZT}$ detectors have non-Gaussian peak shapes and are not symmetric around the peak centers. Detectors that have incomplete charge collection are particularly likely to exhibit peak broadening through low energy tailing.

In an attempt to make their detectors look as good as possible on paper, some manufacturers have created a new measure of peak resolution: 2 x Upper Half Width at Half Maximum "UHWHM". Unfortunately the UHWHM metric does not sufficiently address the issue behind peak resolution - the ability of an analysis program to actually find, de-convolute, and identify peaks. See the following CZT-based figure for an illustration of how 2 x UHWHM can be misleading compared to FWHM.

All of Constellation's resolution specifications are quoted in terms at FWHM.

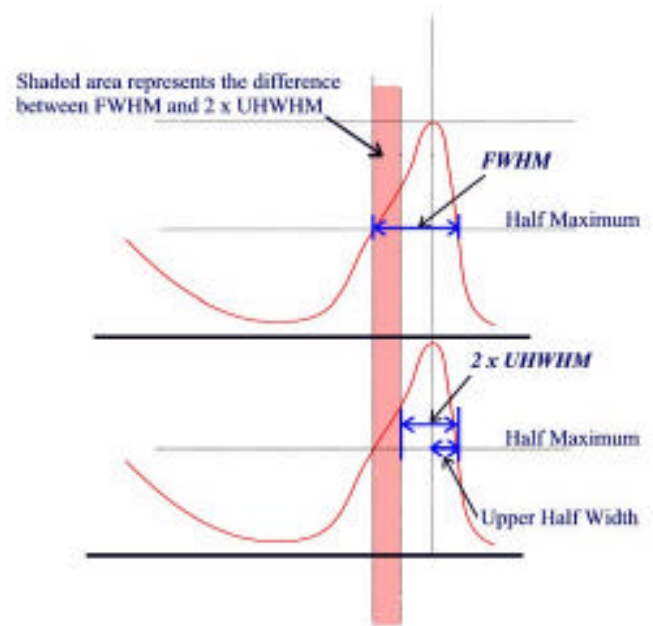




\section{Mercuric Iodide Detectors}

\section{High Density}

The high stopping power of mercuric iodide allows compact, high efficiency detectors. Mercuric iodide has higher $\mathrm{Z}$ and higher density than silicon $(\mathrm{Si})$, germanium $(\mathrm{Ge})$, or CZT.

\begin{tabular}{|l|ll|}
\hline & Z & Density $\left(\mathrm{g} / \mathrm{cm}^{3}\right)$ \\
\hline $\mathrm{Si}$ & 14 & 2.33 \\
$\mathrm{Ge}$ & 32 & 5.32 \\
$\mathrm{CZT}$ & $48-30-52$ & 5.76 \\
$\mathrm{Hgl}_{2}$ & $80-53$ & 6.30 \\
\hline
\end{tabular}

Reference Chart - \% Photopeak Absorption

\begin{tabular}{|l|l|l|l|c|}
\hline Energy & $\mathbf{C Z T}$ & $\mathbf{H g I}_{\mathbf{2}}$ & $\mathbf{\text { NaI }}$ & $\mathbf{G e}$ \\
\hline $70 \mathrm{keV}$ & $97 \%$ & $\mathbf{9 7 \%}$ & $97 \%$ & $90 \%$ \\
\hline $125 \mathrm{keV}$ & $88 \%$ & $\mathbf{9 4 \%}$ & $82 \%$ & $63 \%$ \\
\hline $250 \mathrm{keV}$ & $54 \%$ & $\mathbf{7 5 \%}$ & $54 \%$ & $20 \%$ \\
\hline $500 \mathrm{keV}$ & $19 \%$ & $\mathbf{3 9 \%}$ & $19 \%$ & $4.4 \%$ \\
\hline $700 \mathrm{keV}$ & $10 \%$ & $\mathbf{2 5 \%}$ & $11 \%$ & $2.3 \%$ \\
\hline $2 \mathrm{MeV}$ & $2.3 \%$ & $\mathbf{6 . 3 \%}$ & $2.4 \%$ & $0.5 \%$ \\
\hline
\end{tabular}

\section{Durable and Compact}

Since $\mathrm{HgI}_{2}$ is a solid-state semi-conducting crystal, it does not require a photo-multiplier tube (PMT) for operation. Elimination of a PMT reduces the detection probe size dramatically. With $\mathrm{HgI}_{2}$, portable detection systems can be made that are durable and compact.

Constellation's mercuric iodide designs do not exhibit microphonic problems and are not susceptible to mechanical or thermal shock.

Constellation's portable $\mathrm{HgI}_{2}$ detectors are coated, epoxied and mounted to allow rough handling without microphonic noise.

For example, Constellation's RIC 4000, Radiation Intensity Counter, equipped with a $25 \mathrm{~mm} \times 25 \mathrm{~mm} \times 5 \mathrm{~mm} \mathrm{HgI}{ }_{2}$ detector can be dropped onto a concrete floor from a onemeter height.

\section{Detector Longevity}

Constellation has completed long-term tests to better characterize the long-term performance of $\mathrm{HgI}_{2}$ detectors in the field. These tests are continuing and so far indicate that individual detectors are extremely stable after a year of operation. Stability in this case is judged by the detector's ability to maintain the same spectral resolution over time. This data shows that the FWHM at $59 \mathrm{keV}$ for three of our $\mathrm{HgI}_{2}$ detectors did not change after 2000 hours of operation.

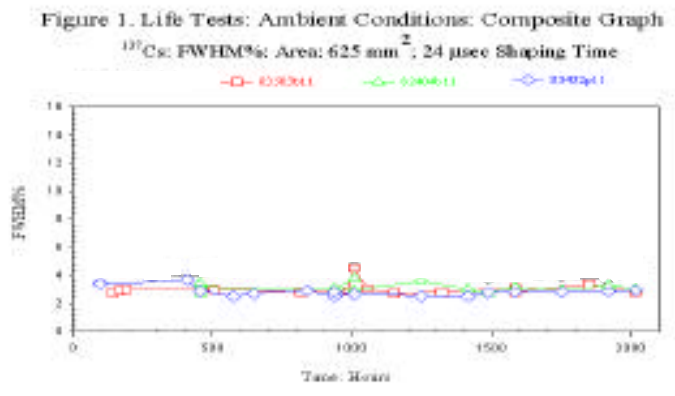

\section{Mercuric lodide at High Temperature}

Not only do $\mathrm{HgI}_{2}$ detectors operate at room temperature with excellent resolution, they also operate at high temperatures with very little loss in resolution. Constellation has tested several detectors at up to $55^{\circ} \mathrm{C}$ with excellent results...

$24{ }^{\circ} \mathrm{C}, 2.9 \%$ FWHM

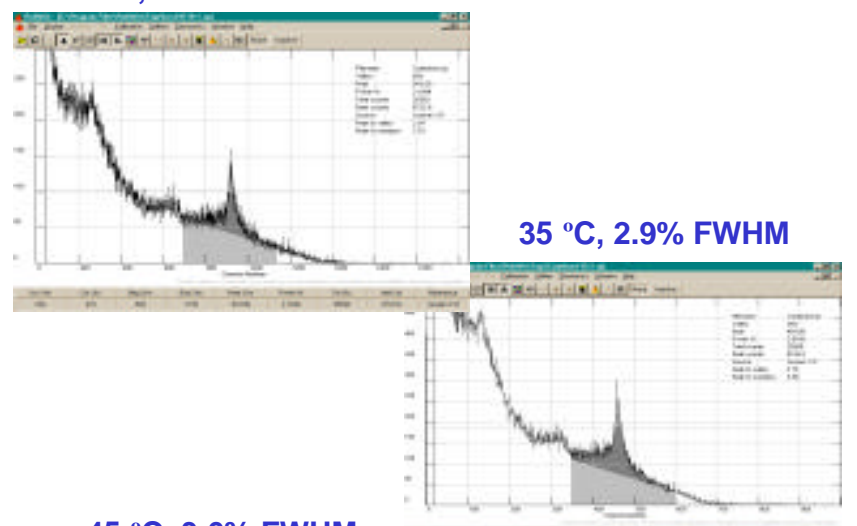

$45{ }^{\circ} \mathrm{C}, 3.6 \%$ FWHM

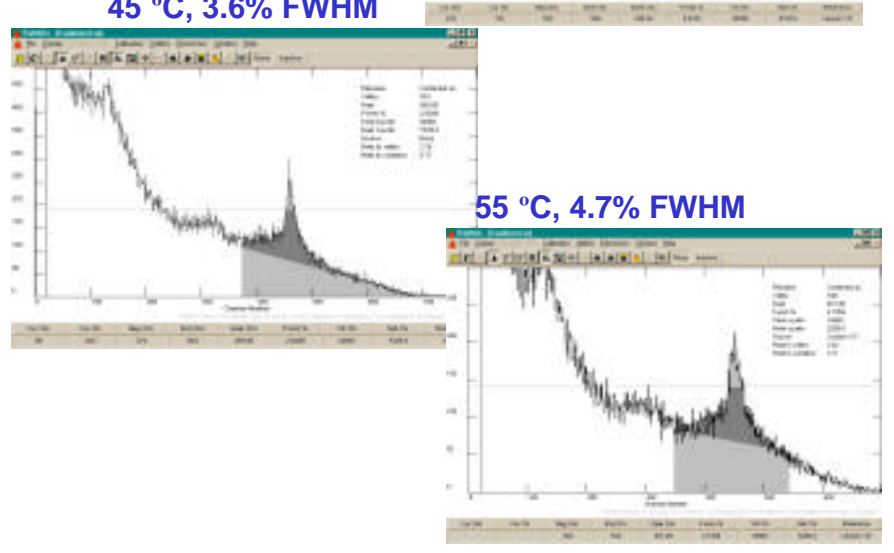




\section{Detector (Crystal) Sizes}

Constellation can fabricate detectors of many different sizes. We generally categorize our detectors into one of three categories, x-ray detectors for spectroscopy, gamma ray detectors for spectroscopy, and $\mathrm{x} /$ gamma ray detectors for counting (no resolution quoted). Our standard detector sizes are:

\begin{tabular}{|c|c|c|}
\hline $\begin{array}{c}\text { X-ray } \\
\text { Spectroscopy } \\
(\mathrm{mm})\end{array}$ & $\begin{array}{c}\text { Gamma Ray } \\
\text { Spectroscopy } \\
(\mathrm{mm})\end{array}$ & $\begin{array}{c}\text { Photon (X/?) } \\
\text { Counters } \\
(\mathrm{mm})\end{array}$ \\
\hline $10 \times 10 \times 1$ & $10 \times 10 \times 1$ & $10 \times 10 \times 1$ \\
\hline $7 \times 7 \times 1$ & $10 \times 10 \times 2$ & $10 \times 10 \times 2$ \\
\hline & $10 \times 10 \times 3$ & $25 \times 25 \times 1$ \\
\hline & $25 \times 25 \times 1$ & $25 \times 25 \times 2$ \\
\hline & $25 \times 25 \times 2$ & $25 \times 25 \times 3$ \\
\hline & $25 \times 25 \times 3$ & $25 \times 25 \times 4$ \\
\hline & & $25 \times 25 \times 5$ \\
\hline
\end{tabular}

Resolution for spectroscopy $\mathrm{x}$-ray detectors is normally quoted in eV FWHM at $5.9 \mathrm{keV}$ (the Fe-55 electron capture $\mathrm{x}$-ray line).

Resolution for spectroscopy gamma ray detectors is normally quoted in \% FWHM at $662 \mathrm{keV}$ (the principle gamma line of ${ }^{137} \mathrm{Cs}$ ) but depending on your application we will also quote at other resolutions such as $122 \mathrm{keV}, 60$ $\mathrm{keV}, 1117 \mathrm{keV}$ and $1333 \mathrm{keV}$.

Custom detector sizes are available. Mercuric iodide probes can be fabricated as small as $1 \mathrm{~mm}^{3}$ or smaller for surgical probes, wound probes, etc. We can also build large areas arrays and stacked arrays of detectors up to $25 \mathrm{~mm} \mathrm{x}$ $25 \mathrm{~mm}$ in area.

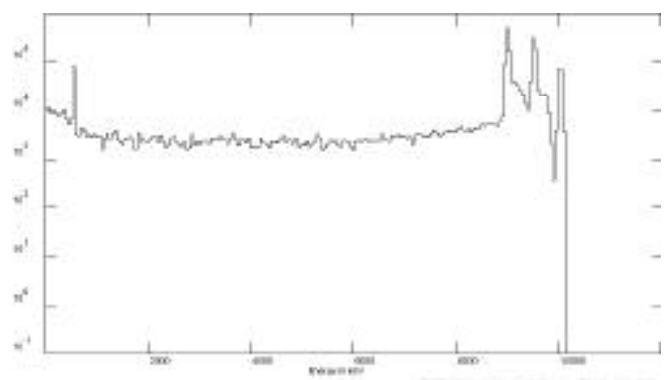

Simulation of 5-element $\mathrm{Hgl}_{2}$ stacked detector each $25 \times 25$ $\mathrm{mm}$ contact area, $2 \mathrm{~mm}$ thick, with $2 \mathrm{~mm}$ inactive (no contacts) detector edges, monoenergetic $10 \mathrm{meV}$ gamma rays uniformly incident as parallel rays

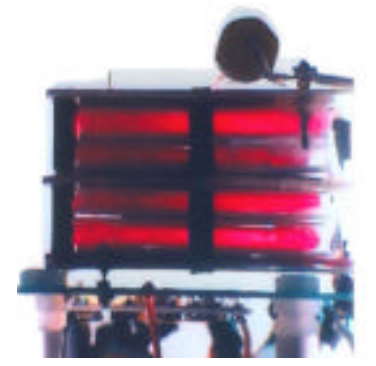

Four-crystal stacked detector.

\section{Mercury Modules}

The Mercury Detector Module contains a mercuric iodide detector and preamplifier and can be configured for a single detector (up to $25 \mathrm{~mm}$ x $25 \mathrm{~mm}$ area), a stacked array, or a position sensitive array. The Mercury module is approximately 3.5 in x 2 in x 2 in $(88.9 \mathrm{~mm} \times 50.8 \mathrm{~mm}$ x $50.8 \mathrm{~mm}$ ) and weighs approximately $0.5 \mathrm{lb}(228 \mathrm{~g})$.

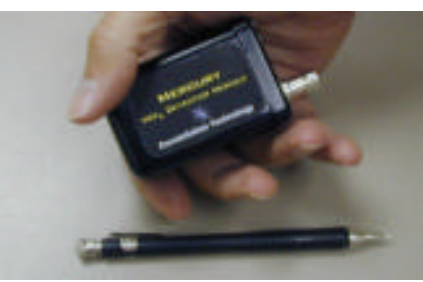

Mercury Module

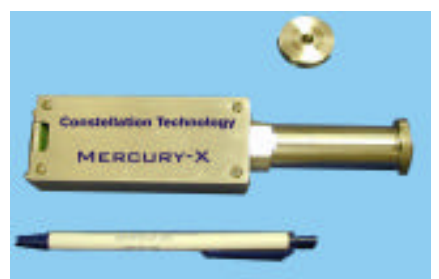

Mercur $y-X$
The Mercury-X is ideal for portable or laboratory x-ray fluorescence experiments and field instruments. It includes the mercuric iodide $\mathrm{x}$-ray detector and preamplifier. The Mercury-X includes a special end cap that allows easy switching between different collimators -- so it's easy to experiment with different collimator aperture sizes and collimator materials. Our standard x-ray detector is $7 \times 7 \mathrm{~mm}$ and about $1 \mathrm{~mm}$ thick. The Mercury-X will also accommodate our larger $10 \mathrm{~mm}$ x $10 \mathrm{~mm}$ photon detectors. 


\section{Appendix D}

Sketch of HgI2 Detector System Submersible Vessel 


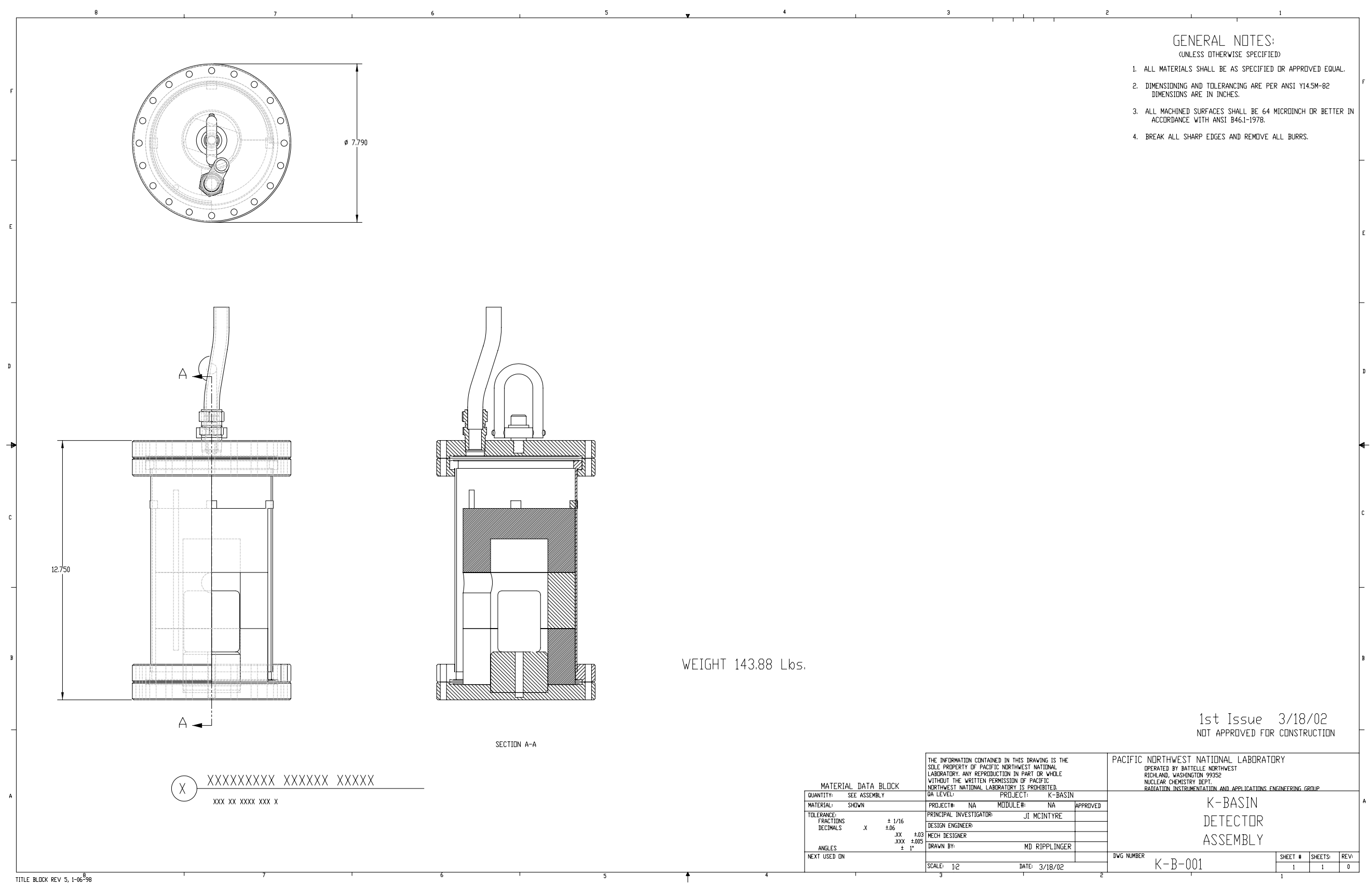




\section{Schlahta, Stephan N}

From: Knight, William J

Sent: Thursday, October 03, 2002 9:05 AM

To: $\quad$ Schlahta, Stephan N

Cc: $\quad$ Milham, Terry W; Waggoner, Larry O

Subject: Radiolytic Degradation of Tygon Tubing.

Subject: ALARA Center Activities for Week of September 23, 2002

... PNNL is going to install a Mercuric lodine detector near the bottom of the fuel pool at $\mathrm{K}$ Basin. They intend to route the two electrical cords through Tygon tubing to keep them dry. The Tygon will be located a few feet from the spent fuel in a very high radiation area. This detector is scheduled to be left in place up to 4 months. PNNL called to find out if the ALARA Center had info on the effects very high radiation would have on the Tygon tubing. Couldn't find out any info and the Tygon company doesn't know either. If anyone who reads this report has information we would appreciate your sharing with us. Please contact Steve Schlahta at (509) 375-6535 if you have info or can recommend a better brand of tubing.

I was able to obtain some information from Rich Chumita, a member of the R\&D department of SaintGobain Performance Plastics, the Manufacturers of Tygon. Mr. Chumita's E-mail to me stated:

"We have no actual test data on our product at high level radiation exposure, but the reference literature states that flexible PVC based products will begin to show a $25 \%$ loss of elongation (hardening or becoming brittle) after 20 Mrads exposure. Which is 20,000,000 Rads (20 X 10E6 Rads.)"

I hope this information helps.

$\mathrm{x}$

\section{William J. Knight}

Health Physicist

233-S Plutonium Concentration Facility Decommissioning Project

200W / MO-409 / 17

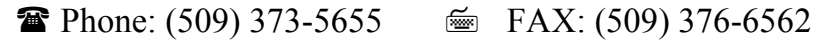

- Pager: 85-5264

埄” E-Mail: william_j_knight@rl.gov 